\title{
Filtrations in Modular Representations of Reductive Lie Algebras
}

\author{
Yiyang Li and Bin Shu
}

\begin{abstract}
Let $G$ be a connected reductive algebraic group over an algebraically closed field $k$ of prime characteristic $p$, and $\mathfrak{g}=\operatorname{Lie}(G)$. In this paper, we study representations of the reductive Lie algebra $\mathfrak{g}$ with $p$-character $\chi$ of standard Levi-form associated with an index subset $I$ of simple roots. With aid of support variety theory we prove a theorem that a $U_{\chi}(\mathfrak{g})$-module is projective if and only if it is a strong "tilting" module, i.e. admitting both $\mathcal{Z}_{Q}$ - and $\mathcal{Z}_{Q}^{w^{I}}$-filtrations (to see Theorem 4.1). Then by analogy of the arguments in [2] for $G_{1} T$-modules, we construct so-called Andersen-Kaneda filtrations associated with each projective $\mathfrak{g}$-module of $p$-character $\chi$, and finally obtain sum formulas from those filtrations.
\end{abstract}

\section{Introduction}

Assume that $k$ is an algebraically closed field of prime characteristic $p$. Let $G$ be a connected reductive algebraic group over $k$, and $\mathfrak{g}=\operatorname{Lie}(G)$. Associated with any given linear form $\chi$ on $\mathfrak{g}, U_{\chi}(\mathfrak{g})$ is defined to be the quotient of the universal enveloping algebra $U(\mathfrak{g})$ by the ideal generated by all $x^{p}-x^{[p]}-\chi(x)^{p}$ with $x \in \mathfrak{g}$. Each class of irreducible representations of $\mathfrak{g}$ correspond to a $p$-character $\chi$ and the representation theory of $\mathfrak{g}$ with this $p$-character is the "union" of the representation theory of $U_{\chi}(\mathfrak{g})$. Furthermore, when we restrict the prime characteristic of the basic fields to the case which we call "very good", a well-known result shows that there is a Morita equivalence between $U_{\chi}(\mathfrak{g})$-module category and $U_{\chi_{n}}(\mathfrak{l})$-module category, where $\mathfrak{l}$ is a certain reductive subalgebra of $\mathfrak{g}$ and $\chi_{n}$ is a so-called nilpotent character of $\mathfrak{l}$ (cf. [16] and [5]). This important result enables us to consider the representations of $U_{\chi}(\mathfrak{g})$ just with nilpotent $\chi$. In the last decade, much progress in modular representations of reductive Lie algebras have been made. Nevertheless, many basic problems remain unsolved.

In this paper, we will focus our concern on the case when $\chi$ is of standard Levi form which is associated with a subset $I$ of simple roots of $\mathfrak{g}$, this means that $\chi$ is regular nilpotent on the Levi factor $\mathfrak{g}_{I}$ for $I$, and is evaluated 0 elsewhere. Owing to the work of Friedlander-Parshall and Jantzen (cf. [5, [10] and [1] ), we have a precise classification of simple $U_{\chi}(\mathfrak{g})$-modules by "highest weights". There are also many good properties in the representations of $U_{\chi}(\mathfrak{g})$ in this case. Especially, one can study the graded module category by modulo $I$, analogous to graded module category in the restricted case, i.e. $\chi=0$ (the graded structure essentially arises from $G_{1} T$-module category in representations of algebraic groups, where $G_{1}$ is the

2000 Mathematics Subject Classification. 17B10; 17B20; 17B35, 17 B50. 
kernel of the first Frobenius homomorphism and $T$ is the maximal torus of $G$ ) (cf. [10, $\S 11]$ and [12, II. $\S 9])$.

Recall that in the BGG theory for complex representations of semi-simple Lie algebras, there is a well-known result that each projective module has a filtration with sub-quotients isomorphic to Verma modules. In the restricted module category of $\mathfrak{g}=\operatorname{Lie}(G)$, Humphreys first proved that each projective module admits an analogous filtration with sub-quotients isomorphic to baby Verma modules $Z(\lambda)$, called a $Z$-filtration (cf. 9]). Furthermore, Cline-Parshall-Scott (CPS for short) in [3] proved that a projective $G_{1} T$-module admits $Z^{w}$-filtratios for an arbitrary given $w$ in the Weyl group where $Z^{w}(\lambda)$ is a twisted baby Verma module. In the same time, CPS also proved that a $G_{1} T$-module is projective if and only if it is a tilting module, i.e. admiting both $Z$-filtration and $Z^{*}$-filtration (or to say: admitting both $Z$ and $Z^{w_{0}}$-filtration for the longest element $w_{0}$ in the Weyl group).

In this paper, we prove a strong version of the above result in the case when $p$ character $\chi$ is of standard Levi form: a $X / \mathbb{Z} I$-graded module of $U_{\chi}(\mathfrak{g})$ is projective if and only if it is a strong "tilting" module, this is to say, it admits both $\mathcal{Z}_{Q}$-filtration and $\mathcal{Z}_{Q}^{\prime}$-filtration, where $\mathcal{Z}_{Q}$ (resp. $\mathcal{Z}_{Q}^{\prime}$ ) are some $\mathfrak{p}_{I}$-induced (resp. $\mathfrak{p}_{I}^{\prime}$-induced) modules from projective covers of the baby Verma modules of $U_{\chi}\left(\mathfrak{g}_{I}\right)$. Here $\mathfrak{p}_{I}$ and $\mathfrak{p}_{I}^{\prime}$ mean respectively positive and negative parabolic subalgebras associated with $I$ (to see Theorem 4.1, where $\mathcal{Z}_{Q}^{\prime}$ has another version $\mathcal{Z}_{Q}^{w^{I}}$ by $w^{I}$-twist which is like $w_{0}$-twist $Z^{w_{0}}$ afore-mentioned). The part of "necessity" in the statement is an implication of Jantzen's result (to see Proposition 2.4). Here, we complete the other part with aid of cohomological support variety theory of restricted Lie algebras.

Consequently, a projective $U_{\chi}(\mathfrak{g})$-module $Q$ is a tilting module 1 , i.e. $Q$ admits both $\mathcal{Z}$-filtration and ${ }^{\tau}\left(\mathcal{Z}^{*}\right)$-filtration where $\tau$ is an involutative automorphism of $\mathfrak{g}$ associated with $I$ (note: ${ }^{\tau}\left(\mathcal{Z}^{*}\right)$ has another version $\mathcal{Z}^{w^{I}}$, to see Lemma 2.6). So, we can adopt the filtration introduced by Andersen and Kaneda in [2], and then obtain the sum formulas (to see Theorems 5.5] and [5.6), which is helpful for us to understand more on simple modules and their character formulas of $U_{\chi}(\mathfrak{g})$. On those characters, Lusztig proposed a hope in [15], which is still unsolved.

In [2], Andersen and Kaneda constructed a filtration associated with each projective $G_{1} T$-module $Q$ from vector spaces $F_{\lambda}(Q)=\operatorname{Hom}_{G_{1} T}\left(Z(\lambda)^{\tau}, Q\right)$, and then proved that the filtration has a sum formula analogous to the Jantzen filtration's, (cf. [2]). We call such a filtration Andersen-Kaneda filtration.

In the case when $\chi$ is of standard Levi-form for reductive Lie algebras, AndersenKaneda filtrations do exist and the corresponding sum formulas can be constructed, by analogy of A-K's arguments with some mild modifications. For the convenience of readers, we complete the arguments.

ACKnowledgement: This work is partially supported by NSF and PCSIRT of China. The authors express thanks to the referee for his/her pointing out some false statements in the original manuscript.

\footnotetext{
${ }^{1}$ The inverse of this statement is not true. The correct inverse statement is just included in Theorem 4.1
} 


\section{Preliminaries}

2.1. Assumptions. Throughout this paper, we always assume that $k$ is an algebraically closed field of prime characteristic $p$. The notations will generally follow 10 .

Let $G$ be a connected and reductive algebraic group over $k$, satisfying the following three hypotheses as in [10, 6.3]:

(H1) The derived group $\mathcal{D} G$ of $G$ is simple connected;

(H2) The prime $p$ is good for $\mathfrak{g}$;

(H3) There exists a $G$-invariant non-degenerate bilinear form on $\mathfrak{g}$,

where $\mathfrak{g}=\operatorname{Lie}(G)$. Those conditions can be explained as follows: when $\mathcal{D} G$ is a simple algebraic group, then the conditions (H1)-(H3) satisfy if and only if $p$ does not divide $n+1$ for type $A_{n} ; p>2$ for types $B_{n}(n \geq 2), C_{n}(n>2)$ and $D_{n}$ $(n \geq 4) ; p>3$ for type $E_{6}, E_{7}, F_{4}$ and $G_{2} ; p>5$ for $E_{8}$.

Fix $T$ a maximal torus of $G$. Let $U(\mathfrak{g})$ be the universal enveloping algebra of $\mathfrak{g}$. For a given $\chi \in \mathfrak{g}^{*}$, set $U_{\chi}(\mathfrak{g})=U(\mathfrak{g}) / J_{\chi}$, a reduced enveloping algebra of $\mathfrak{g}$. Here $J_{\chi}$ is the ideal of $U(\mathfrak{g})$ generated by $x^{p}-x^{[p]}-\chi(x)^{p}$, for all $x \in \mathfrak{g}$. Set $X=X(T)$ the character group of $T$, which is a free abelian group of rank equal to $\operatorname{dim} T$. It contains the subgroup $\mathbb{Z} R$ generated by the root system $R$.

Denote respectively by $R^{ \pm}$the sets of all positive roots and all negative roots. For each $\alpha \in R$, let $\mathfrak{g}_{\alpha}$ denote the subspace of $\mathfrak{g}$ corresponding to $\alpha$ and $\mathfrak{n}^{+}=$ $\sum_{\alpha \in R^{+}} \mathfrak{g}_{\alpha}, \mathfrak{n}^{-}=\sum_{\alpha \in R^{-}} \mathfrak{g}_{\alpha}$. We have the triangular decomposition of $\mathfrak{g}: \mathfrak{g}=\mathfrak{n}^{+} \oplus$ $\mathfrak{h} \oplus \mathfrak{n}^{-}$. Let $\mathfrak{b}^{+}=\mathfrak{h} \oplus \mathfrak{n}^{+}$be the Borel subalgebra of $\mathfrak{g}, \mathfrak{h}$ is the Cartan subalgebra of $\mathfrak{g}$. For each $\alpha \in R$, let $\alpha^{\vee}$ denote the coroot of $\alpha$ and $W$ is the Weyl group generated by all $s_{\alpha}$ with $\alpha \in R$ and $W_{p}$ is the affine Weyl group generated by $s_{\alpha, r p}(r \in \mathbb{Z})$ where $s_{\alpha, r p}, r \in \mathbb{Z}$ are the affine reflection with $s_{\alpha, r p}(\mu)=\mu-\left(\left\langle\mu, \alpha^{\vee}\right\rangle-r p\right) \alpha$. Define $w \cdot \lambda=w(\lambda+\rho)-\rho, w \in W$, the dot action of $w$ on $\lambda$ where $\rho$ is half the sum of all positive roots.

Call $\xi \in \mathfrak{g}^{*}$ nilpotent if $\xi$ is in the coadjoint $G$-orbit of $\chi$ with $\chi\left(\mathfrak{b}^{+}\right)=0$. By Kac-Weisfeiler and Friedlander-Parshall's result, up to Morita equivalence, the study of $U_{\xi}(\mathfrak{g})$-module can be reduced to the study of $U_{\xi_{0}}\left(\mathfrak{g}_{0}\right)$-module for a reductive Lie algebra $\mathfrak{g}_{0}=\operatorname{Lie}\left(G_{0}\right)$ for some connected reductive algebraic group $G_{0}$ satisfying Conditions (H1)-(H3), and nilpotent $\xi_{0} \in \mathfrak{g}_{0}^{*}$. Thus, we only need study the module category of $U_{\chi}(\mathfrak{g})$-modules with $\chi$ 's being nilpotent, up to Morita equivalence. In the whole paper, we always assume that $\chi\left(\mathfrak{b}^{+}\right)=0$, i.e. $\chi$ is nilpotent.

Note that since $\chi\left(\mathfrak{b}^{+}\right)=0$, any simple $U_{0}(\mathfrak{h})$-module is one-dimensional $k_{\lambda}=k$, with $h \cdot 1=\lambda(h)$ for any $h \in \mathfrak{h}$ and $\lambda \in \Lambda:=\left\{\lambda \in \mathfrak{h}^{*} \mid \lambda(h)^{p}=\lambda\left(h^{[p]}\right)\right\}$ which is equal to $X / p X$. And $k_{\lambda}$ can be extended a $U_{0}\left(\mathfrak{b}^{+}\right)$-module with trivial $\mathfrak{n}^{+}$-action. Hence, we have an induced module

$$
Z_{\chi}(\lambda)=U_{\chi}(\mathfrak{g}) \otimes_{U_{0}\left(\mathfrak{b}^{+}\right)} k_{\lambda}
$$

which is called a baby Verma module. Then each simple $U_{\chi}(\mathfrak{g})$ module is the homomorphic image of some baby Verma module $Z_{\chi}(\lambda), \lambda \in \Lambda$.

2.2. Standard Levi forms. We say a $p$-character $\chi$ has standard Levi form if $\chi$ is nilpotent and if there exists a subset $I$ of the set of all simple roots such that 


$$
\chi\left(\mathfrak{g}_{-\alpha}\right)= \begin{cases}\neq 0 & \text { if } \alpha \in I, \\ 0 & \text { if } \alpha \in R^{+} \backslash I .\end{cases}
$$

As in $[10, \S 10.4 ; \S 10.5]$, when $I$ is the full set of all simple roots, we call $\chi$ a regular nilpotent element in $\mathfrak{g}^{*}$. When $I=\{0\}$, we know $U_{\chi}(\mathfrak{g})=U_{0}(\mathfrak{g})$ is the restricted enveloping algebra of $\mathfrak{g}$. We denote $R_{I}$ be the root system corresponding to simple root set $I$ and let $W_{I}$ is the Weyl group generated by all the $s_{\alpha}$ with $\alpha \in I$. Set $w_{I}$ to be the longest element in $W_{I}$ and $w_{0}$ is the longest element in $W$. We denote $w^{I}=w_{I} w_{0}$. Denote $\mathfrak{g}_{I}=\mathfrak{h} \oplus \sum_{\alpha \in R_{I}} \mathfrak{g}_{\alpha} ; \mathfrak{p}=\mathfrak{g}_{I}+\oplus \mathfrak{u}^{+} ;$and $\mathfrak{p}^{\prime}=\mathfrak{g}_{I}+\oplus \mathfrak{u}^{-}$, where $\mathfrak{u}^{+}=\sum_{\alpha \in R^{+} \backslash R_{I}^{+}} \mathfrak{g}_{\alpha}, \mathfrak{u}^{-}=\sum_{\alpha \in R^{+} \backslash R_{I}^{+}} \mathfrak{g}_{-\alpha}$ are the nilpotent radicals of $\mathfrak{p}$ and of $\mathfrak{p}^{\prime}$ respectively.

From now on, we will always assume that $\chi$ is a given $p$-character of standard Levi-form, associated with a subset I of simple roots.

2.3. The category of $X / \mathbb{Z} I$-graded modules. We are going to study certain $X / \mathbb{Z} I$-graded $U_{\chi}(\mathfrak{g})$-module category, denoted by $\mathcal{C}$. It is defined as follows: Each $U_{\chi}(\mathfrak{g})$-module is a direct sum of weight spaces of $\mathfrak{h}$ (note: all weights belong to $X /$ $p X \subset \mathfrak{h}^{*}$ as $\left.\chi(\mathfrak{h})=0\right)$. If $V \in \mathcal{C}$, then each graded component $V_{\lambda+\mathbb{Z} I}$ with $\lambda \in X$ is an $\mathfrak{h}$-submodule, thereby decomposes into weight space for $\mathfrak{h}$. Furthermore, a finitedimensional $X / \mathbb{Z} I$-graded $U_{\chi}(\mathfrak{g})$-module $V$-belongs to $\mathcal{C}$ if and only if all weights of $\mathfrak{h}$ on $V_{\lambda+\mathbb{Z} I}$ have the form $d \mu$ with $\mu \in \lambda+\mathbb{Z} I+p X$ for all $\lambda$. Here $d \lambda$ means the differential of $\lambda \in X(T)$, which satisfys $d \lambda \in \Lambda$.

We call a $U_{\chi}(\mathfrak{g})$-module $M$ gradable if there is on $N \in \mathcal{C}$ such that $M \cong \mathcal{F}(N)$. Here $\mathcal{F}: \mathcal{C} \rightarrow U_{\chi}(\mathfrak{g})$-module category means the forgetful functor.

Lemma 2.1. (cf. [11, 1.4])

(1) Each simple $U_{\chi}(\mathfrak{g})$-module is gradable.

(2) Each baby Verma module is gradable.

(3) Each projective $U_{\chi}(\mathfrak{g})$-module is gradable.

The definition of the $X / \mathbb{Z} I$-graded module category $\mathcal{C}$ can be reformulated (and then extended) as follows.

Let $U=U(\mathfrak{g}) /\left(x_{\alpha}^{p}-\chi\left(x_{\alpha}\right)^{p}, \alpha \in R\right)$, the PBW theorem ensure us the following isomorphism:

$$
U_{\chi}\left(\mathfrak{n}^{-}\right) \otimes U(\mathfrak{h}) \otimes U_{\chi}\left(\mathfrak{n}^{+}\right) \simeq U
$$

We shall denote respectively by $U^{-}, U^{0}$ and $U^{+}$the images of $U_{\chi}\left(\mathfrak{n}^{-}\right), U(\mathfrak{h})$ and $U_{\chi}\left(\mathfrak{n}^{+}\right)$in $U$; these images is respectively isomorphic to $U_{\chi}\left(\mathfrak{n}^{-}\right), U(\mathfrak{h})$ and $U_{\chi}\left(\mathfrak{n}^{+}\right)$. We have a $\mathbb{Z} R / \mathbb{Z} I$-grading on $U$ such that each $\mathfrak{g}_{\alpha}$ with $\alpha \in R \cup\{0\}$ is contained in the homogeneous part of degree $\alpha+\mathbb{Z} I$. We denote by the homogeneous parts of $U$ by $U_{\nu}$ with $\nu \in \mathbb{Z} R / \mathbb{Z} I$.

Let $A$ be a noetherian commutative $k$-algebra. Let

$$
\pi: U^{0} \rightarrow A
$$

denote a $k$-algebra homomorphism. We call a $U \otimes A$-module $M$ to be $\mathfrak{h}$-diagonalizable if

$$
M=\bigoplus_{\phi: \mathfrak{h} \rightarrow A} M^{\phi}, M^{\phi}:=\{m \in M \mid h . m=\phi(h) m\}
$$


In this case, we call $\phi$ an $\mathfrak{h}$-weight of $M$ if $M^{\phi} \neq 0$. Now we can define a category of $U \otimes A$-modules $\mathcal{C}_{A}$. The objects of $\mathcal{C}_{A}$ are $U \otimes A$-modules $M$ which are $\mathfrak{h}$ diagonalizable, together with an $X / \mathbb{Z} I$-grading $M=\bigoplus_{\nu \in X / \mathbb{Z} I} M_{\nu}$ of $M$ satisfying the following conditions:

(A) $M$ is finitely generated over $A$;

(B) $A$ preserves the grading of $M$ :

$$
A M_{\mu+\mathbb{Z I}} \subset M_{\mu+\mathbb{Z} I}
$$

(C) $U_{\nu+\mathbb{Z} I}$ shifts the grading of $M$ by $\nu+\mathbb{Z} I$ :

$$
U_{\nu+\mathbb{Z} I} \cdot M_{\mu+\mathbb{Z} I} \subset M_{\mu+\nu+\mathbb{Z I}}
$$

(D) All $\mathfrak{h}$-weights $\phi$ of $M_{\mu+\mathbb{Z} I}$ have the form:

$$
\phi=\pi+d\left(\mu+\mu^{\prime}\right), \mu^{\prime} \in \mathbb{Z} I .
$$

A morphism between objects in $\mathcal{C}_{A}$ is a $U \otimes A$-homomorphism preserving the gradings. So we can get an induced module in $\mathcal{C}_{A}$

$$
\hat{Z}_{A}(\lambda)=U \otimes_{U^{0} U_{\chi}\left(\mathfrak{n}^{+}\right)} A_{\lambda}
$$

where $A_{\lambda}$ denotes the $\left(\mathfrak{b}^{+}\right) \otimes A$-module $A$ for each $h \in \mathfrak{h}$ acting as multiplication by $\pi(h)+d \lambda(h)$.

We will specialize our choice of the $k$-algebra $A$. When choosing $A=k[t]_{(t)}$ the localization of the polynomial ring $k[t]$ in one variable at the maximal ideal generated by $t$, we will denote by $\tilde{A}$ the fraction field of $k[t]$. The corresponding category of $U \otimes A$-modules and $U \otimes \tilde{A}$-modules will be denoted by $\mathcal{C}_{A}$ and $\mathcal{C}_{\tilde{A}}$. So in the case $A=k[t]_{(t)}, \pi: U^{0} \rightarrow A$ arises from an algebra homomorphism $\pi_{0}: U^{0} \rightarrow k[t]$ which is defined via sending $h_{\alpha}$ to $c_{\alpha} t$ for $c_{\alpha} \in k$ with all $\alpha \in R$ satisfying $c_{w_{I} \alpha}=c_{\alpha}$ and $c_{\alpha} \neq 0$ if and only $\alpha \notin R_{I}$ (cf. [11, $3.1 / 2 / 9$ ] or [14, 13.2]).

When $A=k$, the corresponding category is just $\mathcal{C}$.

In the sequel, we will fix an element $\chi$ in $\mathfrak{g}^{*}$ of standard Levi form, associated with a subset $I$ of the simple root system. Then we may define an order relation $\leq$ on $X / \mathbb{Z} I$ such that $\mu+\mathbb{Z} I \leq \lambda+\mathbb{Z} I$ if and only if there exist integers $m_{\alpha} \geq 0$ with $\lambda-\mu=\sum_{\alpha} m_{\alpha} \alpha+\mathbb{Z} I$. In category $\mathcal{C}$, the baby Verma module is

$$
\hat{Z}_{\chi}(\lambda)=U \otimes_{U^{0} U_{\chi}\left(\mathfrak{n}^{+}\right)} k_{\lambda},
$$

which satisfies $\mathcal{F}\left(\hat{Z}_{\chi}(\lambda)\right) \cong Z_{\chi}(d \lambda)$. When $A^{\prime}=A / t A=k$, we have $\hat{Z}_{A}(\lambda) \otimes_{A} k \simeq$ $\hat{Z}_{\chi}(\lambda)$.

2.4. Twists. (cf. [11, §3.3]) Let $w$ be an element of

$$
W^{I}:=\left\{w \in W \mid w^{-1}(\alpha)>0, \text { for all } \alpha \in I\right\}
$$

We can see that $w^{I} \in W^{I}$. The PBW basis theorem give us an isomorphism

$$
U_{\chi}\left(w \mathfrak{n}^{-}\right) \otimes U^{0} \otimes U_{\chi}\left(w \mathfrak{n}^{+}\right) \simeq U
$$

We can define new categories. In the new category, we get an induced module for $w \in W^{I}$

$$
\hat{Z}_{A}^{w}(\lambda)=U \otimes_{U^{0} U_{\chi}\left(w \mathfrak{n}^{+}\right)} A_{\lambda}
$$

where $A_{\lambda}$ denotes the $\left(w \mathfrak{b}^{+}\right) \otimes A$-module $A$ for each $\mathfrak{h}$ acting as multiplication by $\pi(\mathfrak{h})+(d \lambda)(\mathfrak{h})$. 
When $A=k$, the (twisted) induced module $\hat{Z}_{A}^{w}(\lambda)$ will be denoted by $\hat{Z}_{\chi}^{w}(\lambda)$. We have the following facts.

Lemma 2.2. Let $\lambda, \mu \in X$. Then

(1) (cf. [10, Prop. 11.9]) $\hat{L}_{\chi}(\lambda) \simeq \hat{L}_{\chi}(\mu) \Longleftrightarrow \hat{Z}_{\chi}(\lambda) \simeq \hat{\mathcal{Z}}_{\chi}(\mu) \Longleftrightarrow \mu \in W_{I, p} \cdot \lambda$.

(2) For $w \in W^{I}$ there is an equivalence of categories that takes $\hat{Z}_{\chi}^{w}(\lambda)$ to $\hat{Z}_{w^{-1} \chi}\left(w^{-1} \lambda\right)$.

Proof. (2) Recall that associated with $w \in W$, there is an automorphism of $\mathfrak{g}=\mathfrak{h}+\sum_{\alpha \in R} \mathfrak{g}_{\alpha}$ which stabilizes $\mathfrak{h}$ and makes $\mathfrak{g}_{\alpha}$ into $\mathfrak{g}_{w \alpha}$. We denote it by $\bar{w}$. For $w \in W^{I}$, define $w^{-1} \chi \in \mathfrak{g}^{*}$ via evaluating it $\chi(\bar{w} x)$ at $x$ (similarly, we can define $\left.w^{-1} \lambda \in X\right)$. Under such an automorphism $\bar{w}$ for $w \in W^{I}$, there is an algebra isomorphism between $U_{w^{-1} \chi}(\mathfrak{g})$ and $U_{\chi}(\mathfrak{g})$, which gives rise to an category equivalence between the $X / \mathbb{Z} w I$-graded module category of $U_{w^{-1} \chi}(\mathfrak{g})$ and the $X /$ $\mathbb{Z} I$-graded module category of $U_{\chi}(\mathfrak{g})$, sending $\hat{Z}_{w^{-1} \chi}\left(w^{-1} \lambda\right)$ to $\hat{Z}_{\chi}^{w}(\lambda)$.

2.5. $\mathcal{Z}_{Q}-\mathcal{Z}_{Q}^{w^{I}}$-Filtrations. As in the previous section, we maintain the assumption that the $p$-character $\chi$ is a given standard Levi form, in connection with a subset of simple roots $I=\left\{\alpha \in R \mid \chi\left(x_{-\alpha}\right) \neq 0\right\}$. As the subspaces $\mathfrak{p}$ and $\mathfrak{p}^{\prime}$ of $\mathfrak{g}$ are homogeneous, the algebra $U_{\chi}(\mathfrak{p})$ and $U_{\chi}\left(\mathfrak{p}^{\prime}\right)$ have a natural grading by $X / \mathbb{Z} I$. When we extend a $U_{\chi}\left(\mathfrak{g}_{I}\right)$-module to a $U_{\chi}(\mathfrak{p})$-module or a $U_{\chi}\left(\mathfrak{p}^{\prime}\right)$-module, we will regard $M$ as a graded module with $M_{0}=M$ and $M_{\lambda}=0$ if all $\lambda \neq 0$. The situation for $\mathfrak{p}^{\prime}$ is the same as $\mathfrak{p}$.

For each $U_{\chi}\left(\mathfrak{g}_{I}\right)$-module $M$ set

$$
\mathcal{Z}(M)=U_{\chi}(\mathfrak{g}) \otimes_{U_{\chi}(\mathfrak{p})} M
$$

and

$$
\mathcal{Z}^{\prime}(M)=U_{\chi}(\mathfrak{g}) \otimes_{U_{\chi}\left(\mathfrak{p}^{\prime}\right)} M .
$$

By the arguments in [11, 1.16], we know that both $\mathcal{Z}(M)$ and $\mathcal{Z}^{\prime}(M)$ have natural $X / \mathbb{Z} I$-gradings, identifying $\mathcal{Z}(M)_{0}$ with $M$ as a $U_{\chi}\left(\mathfrak{g}_{I}\right)$-module, $\mathcal{Z}(M)_{\lambda}=0$ implies $\lambda \leq 0$ and $\mathcal{Z}^{\prime}(M)_{\lambda} \neq 0$ implies $\lambda \geq 0$. The corresponding modules in $\mathcal{C}$ are distinguished by wearing a cap, like $\hat{\mathcal{Z}}(M)$ and $\hat{\mathcal{Z}}^{\prime}(M)$.

Consider $Z_{\chi, I}(\lambda)=U_{\chi}\left(\mathfrak{g}_{I}\right) \otimes_{U_{\chi}\left(\mathfrak{g}_{I} \cap \mathfrak{b}^{+}\right)} k_{\lambda}, \lambda \in \Lambda_{\chi}$. Then $\left.\chi\right|_{\mathfrak{g}_{I}}$ is regular nilpotent. By [5, 4.2/3], we know it's a simple $U_{\chi}\left(\mathfrak{g}_{I}\right)$ module (also refer to [10, $\S 10$ and $\left.\S 11\right]$ ). Let $Q_{\chi, I}(\lambda)$ be the projective cover of the $U_{\chi}\left(\mathfrak{g}_{I}\right)$-module $Z_{\chi, I}(\lambda)$. Thus, we have induced modules of $\mathfrak{g}$ :

$$
\mathcal{Z}\left(Z_{\chi, I}(\lambda)\right) \cong Z_{\chi}(\lambda)
$$

and

$$
\mathcal{Z}\left(Q_{\chi, I}(\lambda)\right)
$$

We denote both by $\mathcal{Z}(\lambda)$ and $\mathcal{Z}(Q, \lambda)$ respectively. By Lemma 2.1, there are corresponding modules in $\mathcal{C}$, denoted by $\hat{\mathcal{Z}}(\lambda)$ and $\hat{\mathcal{Z}}(Q, \lambda)$.

Since $Q_{\chi, I}(\lambda)$ has a filtration of length $\left|W_{I} \cdot \bar{\lambda}\right|$ with all quotient of subsequent terms in the filtration isomorphic to $Z_{\chi, I}(\lambda)$ (cf. [10, $\left.\left.\S 10.10\right]\right), \mathcal{Z}\left(Q_{\chi, I}(\lambda)\right.$ ) has a filtration of length $\left|W_{I} \cdot \bar{\lambda}\right|$ with each quotient of subsequent terms in the filtration isomorphic to $Z_{\chi}(\lambda)$. Here and further, $\bar{\lambda}$ stands for the image of $\lambda$ in $X / p X$.

Denoting by $\hat{Q}_{\chi}(\lambda)$ the projective cover of the simple module $\hat{L}_{\chi}(\lambda)$, we know that $\hat{Q}_{\chi}(\lambda)$ has a filtration with each quotient of subsequent terms isomorphic to $\hat{\mathcal{Z}}(Q, \mu)$ for some $\mu \in X$, while the number of factors isomorphic to a given $\hat{\mathcal{Z}}(Q, \mu)$ is 
equal to $\left[\hat{Z}_{\chi}(\mu): \hat{L}_{\chi}(\lambda)\right]$ (cf. [10, Proposition 10.11]). Consequently, the projective module $\hat{Q}_{\chi}(\lambda)$ has a filtration where all factors of subsequent terms are isomorphic to $\hat{Z}_{\chi}(\lambda)$ for some $\lambda \in X$, The number (denoted by $\left(\hat{Q}_{\chi}(\lambda): \hat{Z}_{\chi}(\mu)\right)$ ) of factors in such a filtration of $\hat{Q}_{\chi}(\lambda)$ isomorphic to a given $\hat{Z}_{\chi}(\mu)$ is equal to $\left|W_{I} \cdot \bar{\mu}\right| \cdot\left[\hat{Z}_{\chi}(\mu): \hat{L}_{\chi}(\lambda)\right]$.

Recall that $\mathfrak{g}_{I}=\mathfrak{h} \oplus \sum_{\alpha \in R_{I}} \mathfrak{g}_{\alpha} ; \mathfrak{p}=\mathfrak{g}_{I} \oplus \mathfrak{u}^{+}$. Then $w^{I}(\mathfrak{p})=\mathfrak{p}^{\prime}=\mathfrak{g}_{I} \oplus$ $\sum_{\alpha \notin w^{I}(\mathbb{Z} I)} \mathfrak{g}_{-\alpha}$. Actually, $w^{I}(I)=-w_{I}(I) \in \mathbb{Z} I$, and $w^{I}(\alpha)=w_{0}(\alpha)$ for $\alpha \in$ $R^{+} \backslash R_{I}^{+}$, and then $w^{I}\left(\mathfrak{g}_{I}\right)=\mathfrak{g}_{I}$. Hence we can define the twist induced modules as follows:

and

$$
\mathcal{Z}^{\prime}\left(Z_{\chi, I}(\lambda)\right)=U_{\chi}(\mathfrak{g}) \otimes_{U_{\chi}\left(w^{I}(\mathfrak{p})\right)} Z_{\chi, I}(\lambda) \cong Z_{\chi}^{w^{I}}(\lambda) .
$$

$$
\mathcal{Z}^{\prime}\left(Q_{\chi, I}(\lambda)\right)=U_{\chi}(\mathfrak{g}) \otimes_{U_{\chi}\left(w^{I}(\mathfrak{p})\right)} Q_{\chi, I}(\lambda) .
$$

Both of them will be denoted by $\mathcal{Z}^{w^{I}}(\lambda)$ and $\mathcal{Z}^{w^{I}}(Q, \lambda)$ respectively. The corresponding graded modules in $\mathcal{C}$ are denoted by $\hat{\mathcal{Z}}^{w^{I}}(\lambda)$ and $\hat{\mathcal{Z}}^{w^{I}}(Q, \lambda)$.

Definition 2.3. $\quad(1)$ Let $M \in \mathcal{C} . M$ is said to has a $\hat{\mathcal{Z}}$-filtration (resp. $\hat{\mathcal{Z}}^{w^{I}}$ filtration) if there is a filtration with each quotient of subsequent terms in the filtration isomorphic to $\hat{\mathcal{Z}}_{\chi}(\mu)$ (resp. $\hat{\mathcal{Z}}_{\chi}^{w^{I}}(\mu)$ for some $\mu \in X$.

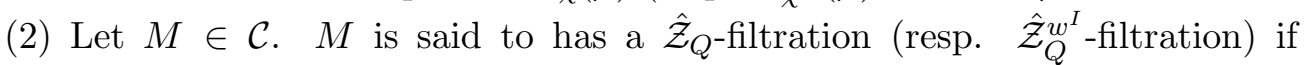
there is a filtration with each quotient of subsequent terms in the filtration isomorphic to $\hat{\mathcal{Z}}(Q, \mu)$ (resp. $\left.\hat{\mathcal{Z}}^{w^{I}}(Q, \mu)\right)$ for some $\mu \in X$.

(Note: the same notions in the category $\mathcal{C}_{A}$ can be defined, in the same sense of (1) and (2) respectively.)

One of Jantzen's results [11, Proposition 2.1] implies the following proposition.

Proposition 2.4. Let $\chi \in \mathfrak{g}^{*}$ be of standard Levi form. If the object $M$ in the category $\mathcal{C}$ is projective, then $M$ has both $\hat{\mathcal{Z}}_{Q}$-filtration and $\hat{\mathcal{Z}}_{Q}^{\omega^{I}}$-filtration, thereby has both a $\hat{\mathcal{Z}}_{\chi}$-filtration and a $\hat{\mathcal{Z}}_{\chi}^{w^{I}}$-filtration.

Proof. Assume $M \in \mathcal{C}$ is projective, then it's a projective $U_{\chi}(\mathfrak{p})$-module. By [11, Proposition 2.1], $M$ has a $\hat{\mathcal{Z}}_{Q}$-filtration. Symmetrically, $M$ has a $\hat{\mathcal{Z}}_{Q}^{w^{I}}$-filtration.

Remark 2.5. (1) Generally speaking, it's no longer true that $M$ in $\mathcal{C}$ is projective if $M$ admits both $\hat{\mathcal{Z}}_{\chi^{-}}$and $\hat{\mathcal{Z}}_{\chi}^{w^{I}}$-filtrations. An obvious counter-example is $M=\hat{Z}_{\chi}(\lambda)$ for a regular nilpotent $\chi$. In such a case, $I$ is just the whole simple roots, and a baby Verma module coincides with its $w^{I}$-twist. However, $M$ is projective only when $\lambda$ is a Steinberg weight (cf. [8]).

(2) By the same argument as [1, 4.19], we have the following basic facts:

(i) There is a unique projective module up to isomorphism, $\hat{Q}_{A}(\lambda) \in \mathcal{C}_{A}$ satisfying $\hat{Q}_{A}(\lambda) \otimes_{A} k \cong \hat{Q}_{\chi}(\lambda)$, and $\left(\hat{Q}_{A}(\lambda): \hat{Z}_{A}(\mu)\right)=\left(\hat{Q}_{\chi}(\lambda)\right.$ : $\left.\hat{Z}_{\chi}(\mu)\right)$.

(ii) By the argument in 92.5 (i) gives us $\left(\hat{Q}_{A}(\lambda): \hat{Z}_{A}(\mu)\right)=\left|W_{I} \cdot \bar{\mu}\right|$. $\left[\hat{Z}_{\chi}(\mu): \hat{L}_{\chi}(\lambda)\right]$

(iii) Any projective module $\hat{Q}$ in $\mathcal{C}_{A}$ is isomorphic to a direct sum of certain $\hat{Q}_{A}(\lambda)$. Thus, we easily know that the rank of the free $A$-module $\operatorname{Hom}_{\mathcal{C}_{A}}\left(\hat{Q}, \hat{Z}_{A}(\mu)\right)$ equal to $\left(\hat{Q}: \hat{Z}_{A}(\mu)\right)$. 
2.6. Duality. (cf. [10, $\S 11.4 ; \S 11.5 ; \S 11.16]$ ) Jantzen constructed a duality $\tau\left(-^{*}\right)$ on the category $\mathcal{C}$. By [11, 1.14], there is an automorphism $\tau$ of $G$ satisfying $\tau(T)=T$, with derivative that acts in the following way:

$$
\begin{aligned}
& \tau\left(x_{\alpha}\right)=x_{-w_{I} \alpha .} \\
& \tau\left(h_{\alpha}\right)=h_{-w_{I} \alpha .} .
\end{aligned}
$$

Note that $\tau^{2}=\mathbf{i d}$. It has the properties that $\chi \circ \tau^{-1}=-\chi$ and $\lambda \circ \tau^{-1}=-w_{I}(\lambda)$ for all $\lambda \in X$. In category $\mathcal{C}$, we have $[1, \S 11.6]$

Lemma 2.6. Let $\mu \in X$.

$$
\hat{Z}_{\chi}(\mu) \simeq{ }^{\tau}\left(\hat{Z}_{\chi}^{w^{I}}\left(\mu^{w^{I}}\right)^{*}\right), \text { where } \mu^{w^{I}}=\mu-(p-1)\left(\rho-w^{I} \rho\right) .
$$

Here $\rho$ is half the sum of the positive roots of $R$.

Remark 2.7. The $\tau$-duality can be extended to the category $\mathcal{C}_{A}$. Let $\mathrm{M}$ be an object of $\mathcal{C}_{A}$. Define ${ }^{\tau} M$ to be $\operatorname{Hom}_{A}(M, A)$, as an $A$-module. The $U$-action on $\tau(M)$ is defined by:

$$
u \in \mathfrak{g},(u . f)(m):=f\left(-\tau^{-1}(u) . m\right), m \in M .
$$

In general, if $M \in \mathcal{C}_{A}$ is a free $A$-module,then ${ }^{\tau}\left({ }^{\tau} M\right) \cong M$. For another $A$-free module $M^{\prime} \in \mathcal{C}_{A}, \operatorname{Hom}_{\mathcal{C}_{A}}\left(M, M^{\prime}\right) \cong \operatorname{Hom}_{\tau} \mathcal{C}_{A}\left({ }^{\tau} M^{\prime},{ }^{\tau} M\right)$ as $A$-free modules (compare $[2, \S 1.6])$. Here ${ }^{\tau} \mathcal{C}_{A}$ means an version of the module category corresponding to $-\chi$, parallel to $\mathcal{C}_{A}$.

In category $\mathcal{C}_{A}$, one readily has an analogy of Lemma 2.6 by a natural way (cf. [14, §13.6]):

Lemma 2.8. Let $\mu \in X$.

$$
\hat{Z}_{A}(\mu) \simeq{ }^{\tau}\left(\hat{Z}_{A}^{w^{I}}\left(\mu^{w^{I}}\right)^{*}\right), \text { where } \mu^{w^{I}}=\mu-(p-1)\left(\rho-w^{I} \rho\right) .
$$

Here $\rho$ is half the sum of the positive roots of $R$.

\section{Baby Verma modules and their twists}

In this section, we will give some computation on hom-spaces and extensions between (baby) Verma modules and their twists in the module category $\mathcal{C}_{A}$, which will be used later. Before that, we first prove some general formulation of Lemma 2.2 which will be the start point of our argument in the sequel.

Lemma 3.1. Let $\lambda, \mu \in X$. Then $\operatorname{Hom}_{\mathcal{C}_{A}}\left(\hat{Z}_{A}(\lambda), \hat{Z}_{A}(\mu)\right) \simeq A \Longleftrightarrow \mu \in W_{I, p} \cdot \lambda$.

Proof. First, we assert that all $\hat{Z}_{\tilde{A}}(\lambda)=\hat{Z}_{A}(\lambda) \otimes_{A} \tilde{A}$ are simple in $\mathcal{C}_{\tilde{A}}$. We will prove this by standard argument. Consider $\mathfrak{g}_{\bar{K}}=\mathfrak{g}_{K} \otimes_{K} \bar{K}$, where $K=\tilde{A}, \mathfrak{g}_{K}$ the extension of Lie algebra $\mathfrak{g}$ by field extension of $K / k$ and $\bar{K}$ is the algebraically closure of $K$. Then we have

$$
\hat{Z}_{\bar{K}}(\lambda) \cong \hat{Z}_{\chi_{\pi}}(\lambda)
$$

where $\chi_{\pi} \in \mathfrak{g}_{\bar{K}}^{*}$ is defined via $\chi_{\pi}\left(h_{\alpha}\right)=c_{\alpha} t-\left(c_{\alpha} t\right)^{\frac{1}{p}} \neq 0$ if $\alpha \in R \backslash R_{I}, \chi_{\pi}\left(h_{\alpha}\right)=$ 0 if $\alpha \in R_{I}$ and $\left.\chi_{\pi}\right|_{\mathfrak{n}^{ \pm}}=\left.\chi\right|_{\mathfrak{n}^{ \pm}}$. Here $c_{\alpha}$ is defined as in 2.3 . Then $\chi_{\pi}$ has a Chevalley-Jordan decomposition $\chi_{\pi, s}+\chi_{\pi, n}$ where $\chi_{\pi, s} \in \mathfrak{g}_{\bar{K}}^{*}$ is the trivial extension of $\left.\chi_{\pi}\right|_{\mathfrak{h}} \in \mathfrak{h}^{*} \subset \mathfrak{g}_{\bar{K}}^{*}$, and $\chi_{\pi, n} \in \mathfrak{g}_{\bar{K}}^{*}$ is the trivial extension of $\chi \in \mathfrak{g}^{*} \subset \mathfrak{g}_{\bar{K}}^{*}$. Thus the centralizer $\mathfrak{c}_{\mathfrak{g}_{\bar{K}}}\left(\chi_{\pi}\right)$ of $\chi_{\pi}$ in $\mathfrak{g}_{\bar{K}}$ coincides with $\mathfrak{g}_{I} \otimes \bar{K}$. By [16, 2.4] (more precisely 
[5. 3.2 and 8.5]), $U_{\chi_{\pi}}\left(\mathfrak{g}_{\bar{K}}\right)$ is Morita equivalent to $U_{\chi}\left(\mathfrak{g}_{I} \otimes \bar{K}\right)$, the latter of which is of standard Levi form. All Verma modules of $U_{\chi}\left(\mathfrak{g}_{I} \otimes \bar{K}\right)$ are simple, which implies the corresponding baby Verma modules $U_{\chi_{\pi}}(\lambda)$ of $U_{\chi_{\pi}}\left(\mathfrak{g}_{\bar{K}}\right)$ are simple, under the Morita equivalence. So $\hat{Z}_{\bar{K}}(\lambda)$ is simple (notice (3.1)). From $\hat{Z}_{\bar{K}}(\lambda) \cong \hat{Z}_{\tilde{A}}(\lambda) \otimes_{\tilde{A}} \bar{K}$, it follows that all $\hat{Z}_{\tilde{A}}(\lambda)$ are simple.

Furthermore, by the Morita equivalence and Lemma 2.2 (over $\bar{K}$ ), we know

$$
\operatorname{Hom}_{\mathcal{C}_{\tilde{A}}}\left(\hat{Z}_{\tilde{A}}(\lambda), \hat{Z}_{\tilde{A}}(\mu)\right)= \begin{cases}\tilde{A} & \text { if } \lambda \in W_{I, p} \cdot \mu, \\ 0 & \text { otherwise. }\end{cases}
$$

Observe that $\tilde{A}$ is $A$-flat, and the $A$-free module $\operatorname{Hom}_{\mathcal{C}_{A}}\left(\hat{Z}_{A}(\lambda), \hat{Z}_{A}(\mu)\right)$ satisfies by [4, 2.38]

$$
\operatorname{Hom}_{\mathcal{C}_{A}}\left(\hat{Z}_{A}(\lambda), \hat{Z}_{A}(\mu)\right) \otimes_{A} \tilde{A} \cong \operatorname{Hom}_{\mathcal{C}_{\tilde{A}}}\left(\hat{Z}_{\tilde{A}}(\lambda), \hat{Z}_{\tilde{A}}(\mu)\right) .
$$

Hence $\operatorname{Hom}_{\mathcal{C}_{A}}\left(\hat{Z}_{A}(\lambda), \hat{Z}_{A}(\mu)\right)=A$ if and only if $\lambda \in W_{I, p} \cdot \mu$.

The following lemmas are crucially useful in the last section.

Lemma 3.2. (1) Let $\lambda \in X$, and $\lambda^{\sigma}=\lambda+(p-1)(\sigma \rho-\rho)$ for $\sigma \in W^{I}$. Then for $w, w^{\prime} \in W^{I}, \operatorname{Hom}_{\mathcal{C}_{A}}\left(Z_{A}^{w}\left(\lambda^{w}\right), Z_{A}^{w^{\prime}}\left(\lambda^{w^{\prime}}\right)\right) \cong A$.

(2) In particular, for $\lambda \in X$, and $w^{I}=w_{I} w_{0}$,

$$
\operatorname{Hom}_{\mathcal{C}_{A}}\left(\hat{Z}_{A}(\lambda), \hat{Z}_{A}^{w^{I}}\left(\lambda^{w^{I}}\right)\right) \simeq A
$$

and

$$
\operatorname{Hom}_{\mathcal{C}_{A}}\left(\hat{Z}_{A}^{w^{I}}\left(\lambda^{w^{I}}\right), \hat{Z}_{A}(\lambda)\right) \simeq A .
$$

Proof. We can prove the lemma, following the argument in the proof of $\mathbf{1}$, 4.7] (or to see [2, 1.7]).

We have the following reformulation of Lemma 3.2, dealing with general situations.

Lemma 3.3. Let $\lambda, \mu \in X$. For any $w \in W^{I}$,

$$
\operatorname{Hom}_{\mathcal{C}_{A}}\left(\hat{Z}_{A}^{w}\left(\lambda^{w}\right), \hat{Z}_{A}(\mu)\right) \simeq \begin{cases}A & \text { if } \mu \in W_{I, p} \cdot \lambda \\ 0 & \text { others }\end{cases}
$$

Proof. As $\tilde{A}$ is $A$-flat, applying [4, 2.38] we know that

$$
\operatorname{Hom}_{\mathcal{C}_{A}}\left(\hat{Z}_{A}^{w}\left(\lambda^{w}\right), \hat{Z}_{A}(\mu)\right) \otimes_{A} \tilde{A} \cong \operatorname{Hom}_{\mathcal{C}_{\tilde{A}}}\left(\hat{Z}_{\tilde{A}}^{w}\left(\lambda^{w}\right), \hat{Z}_{\tilde{A}}(\mu)\right) .
$$

If $\operatorname{Hom}_{\mathcal{C}_{A}}\left(\hat{Z}_{A}^{w}\left(\lambda^{w}\right), \hat{Z}_{A}(\mu)\right)$ is nonzero, then $\operatorname{Hom}_{\mathcal{C}_{\tilde{A}}}\left(\hat{Z}_{\tilde{A}}^{w}\left(\lambda^{w}\right), \hat{Z}_{\tilde{A}}(\mu)\right)$ is nonzero. By the argument in the proof of Lemma 3.1, we know that both $\hat{Z}_{\tilde{A}}^{w}\left(\lambda^{w}\right)$ and $\hat{Z}_{\tilde{A}}(\mu)$ are simple. So $\hat{Z}_{\tilde{A}}^{w}\left(\lambda^{w}\right) \cong \hat{Z}_{\tilde{A}}(\mu)$. Lemma $\left[3.2(1)\right.$ tells us that $\hat{Z}_{\tilde{A}}^{w}\left(\lambda^{w}\right) \cong \hat{Z}_{\tilde{A}}(\lambda)$. Using Lemma 2.2, we finally obtain $\mu \in W_{I, p} \cdot \lambda$. Conversely, if $\mu \in W_{I, p} \cdot \lambda$, by the above argument we know $\operatorname{Hom}_{\mathcal{C}_{A}}\left(\hat{Z}_{A}^{w}\left(\lambda^{w}\right), \hat{Z}_{A}(\mu)\right) \otimes_{A} \tilde{A} \cong \tilde{A}$. Note that the first term in the tensor product is a free-A module of finite rank, thereby of rank one. We complete the proof.

Choose a reduced expression $w^{I}=s_{1} s_{2} \cdots s_{N}$ where $s_{i}=s_{\alpha_{i}}$ for some simple roots $\alpha_{i}$. Set $w_{i}=s_{1} \cdots s_{i-1}, i=1, \cdots, N+1$ with convention $w_{1}=1$. Then all $w_{i} \alpha_{i}$ are distinct, they are exactly the positive roots made negative by $w_{0} w_{I}=\left(w^{I}\right)^{-1}$, constituting $R^{+} \backslash R_{I}^{+}$. Furthermore, the positive roots made negative by $w_{i}^{-1}$ are 
exactly $w_{1} \alpha_{1}, \cdots, w_{i-1} \alpha_{i-1}$. This shows that $w_{i}^{-1}(I) \subset R^{+}$, thereby $w_{i} \in W^{I}$. Hence we can construct the $w_{i}$-twist baby Verma modules $Z_{A}^{w_{i}}(-)$ and $Z_{\chi}^{w_{i}}(-)$. Set

$$
\hat{Z}_{A}^{i}=Z_{A}^{w_{i}}\left(\lambda^{w_{i}}\right) \quad\left(\operatorname{resp} . \hat{Z}_{\chi}^{i}=Z_{\chi}^{w_{i}}\left(\lambda^{w_{i}}\right)\right) .
$$

Then $\hat{Z}_{A}^{1}=\hat{Z}_{A}(\lambda)$ and $\hat{Z}_{A}^{N+1}=Z_{A}^{w^{I}}\left(\lambda^{w^{I}}\right)$. By Lemma 3.2(1), we can take a generator $\varphi_{i} \in \operatorname{Hom}_{\mathcal{C}_{A}}\left(\hat{Z}^{i}, \hat{Z}^{i+1}\right) \cong A$, which is unique up to units in $A$. Similarly, we take a generator $\varphi_{i}^{\prime} \in \operatorname{Hom}_{\mathcal{C}_{A}}\left(\hat{Z}_{A}^{i+1}, \hat{Z}_{A}^{i}\right) \cong A$. Precisely, those module homomorphisms $\varphi_{i}$ and $\varphi_{i}^{\prime}$ can be taken respectively via: sending $1 \otimes 1$ to $x_{w_{i} \alpha_{i}}^{p-1} \otimes 1$, and sending $1 \otimes 1$ to $x_{-w_{i} \alpha_{i}}^{p-1} \otimes 1$.

By change of rings $-\otimes_{A} k$ (note that $k \cong A / t A$ ), one has extensions of both $\varphi_{i}$ and $\varphi_{i}^{\prime}: \bar{\varphi}_{i}=\varphi_{i} \otimes_{A} k$ and $\bar{\varphi}_{i}^{\prime}=\varphi_{i}^{\prime} \otimes_{A} k$ in $\operatorname{Hom}_{\mathcal{C}}\left(Z_{\chi}^{i}, Z_{\chi}^{i+1}\right)$ and $\operatorname{Hom}_{\mathcal{C}}\left(Z_{\chi}^{i+1}, Z_{\chi}^{i}\right)$ respectively. It can be known from the forthcoming lemma that if $\left\langle\lambda+\rho, w_{i} \alpha_{i}^{\vee}\right\rangle \equiv 0$ $\bmod (p)$, then both $\bar{\varphi}_{i}$ and $\bar{\varphi}_{i}^{\prime}$ are isomorphisms in $\mathcal{C}$.

Lemma 3.4. Up to units in $A$ the following statements hold

$\begin{aligned} \text { (1) } \varphi_{i} \circ \varphi_{i}^{\prime} & = \begin{cases}t \mathbf{i d}_{Z_{A}^{i}}, & \text { if }\left\langle\lambda+\rho, w_{i} \alpha_{i}^{\vee}\right\rangle \neq 0 \bmod (p), \\ \operatorname{id}_{Z_{A}^{i}}, & \text { otherwise } .\end{cases} \\ \text { (2) } \varphi_{i}^{\prime} \circ \varphi_{i} & = \begin{cases}t \mathbf{i d}_{Z_{A}^{i+1}}, & \text { if }\left\langle\lambda+\rho, w_{i} \alpha_{i}^{\vee}\right\rangle \neq 0 \bmod (p), \\ \operatorname{id}_{Z_{A}^{i+1}}, & \text { otherwise. }\end{cases} \end{aligned}$

(3) If $\left\langle\lambda+\rho, w_{i} \alpha_{i}^{\vee}\right\rangle^{A} \equiv 0 \bmod (p)$, then both $\varphi_{i}$ and $\varphi_{i}^{\prime}$ are isomorphisms.

Proof. (1) Thanks to Lemma 3.2, it is sufficient to evaluate $\varphi_{i}^{\prime} \circ \varphi_{i}$ on a single non-zero element $v$ in $Z_{A}^{i}$, say $v_{0}=1 \otimes 1$. Recall that for $0 \leq s \leq p-1, v_{s}:=$ $\frac{x_{-w_{i} \alpha_{i}}^{s}}{s !} \otimes 1 \in Z_{A}^{i}$ and $v_{s}^{\prime}:=\frac{x_{w_{i} \alpha_{i}}^{s}}{s !} \otimes 1 \in Z_{A}^{i+1}$, we have

$$
x_{w_{i} \alpha_{i}} v_{s}=\left(\pi\left(h_{w_{i} \alpha_{i}}\right)+\left\langle\lambda+\rho, w_{i} \alpha_{i}^{\vee}\right\rangle-s\right) v_{s-1},
$$

where $\alpha_{i}^{\vee}$ is the coroot. Note that $\varphi_{i}\left(v_{0}\right)=(p-1) ! v_{p-1}^{\prime}$ and $\varphi_{i}^{\prime}\left(v_{0}^{\prime}\right)=(p-1) ! v_{p-1}$. Hence we have

$$
\begin{aligned}
\varphi_{i}^{\prime} \circ \varphi_{i}\left(v_{0}\right) & =(p-1) ! \prod_{j=1}^{p-1}\left(\pi\left(h_{w_{i} \alpha_{i}}\right)+\left\langle\lambda+\rho, w_{i} \alpha_{i}^{\vee}\right\rangle+j\right) v_{0} \\
& =(p-1) ! \prod_{j=1}^{p-1}\left(c_{w_{i} \alpha_{i}} t+\left\langle\lambda+\rho, w_{i} \alpha_{i}^{\vee}\right\rangle+j\right) v_{0} .
\end{aligned}
$$

When $\left\langle\lambda+\rho, w_{i} \alpha_{i}^{\vee}\right\rangle \equiv 0 \bmod (p)$, the coefficient above is a unit in $A$. When $\left\langle\lambda+\rho, w_{i} \alpha_{i}^{\vee}\right\rangle \neq 0 \bmod (p)$, the coefficient above is equal to $t u$ for a unit $u$ in $A$. Hence, we get the first statement.

The proof of (2) is similar. The third statment is an immediate consequence of (1) and (2).

Remark 3.5. We have general formulas in connection with (3.2) which will be used:

$$
\varphi_{i}\left(v_{s}\right)=(-1)^{s} \frac{(p-1) !}{s !} \prod_{j=1}^{s}\left(\pi\left(h_{w_{i} \alpha_{i}}\right)+\left\langle\lambda+\rho, w_{i} \alpha_{i}^{\vee}\right\rangle-j\right) v_{p-1-s}^{\prime}
$$

and

$$
\varphi_{i}^{\prime}\left(v_{s}^{\prime}\right)=\frac{(p-1) !}{s !} \prod_{j=1}^{s}\left(\pi\left(h_{w_{i} \alpha_{i}}\right)+\left\langle\lambda+\rho, w_{i} \alpha_{i}^{\vee}\right\rangle+j\right) v_{p-1-s}
$$


We can take the generator $\varpi$ of $\operatorname{Hom}_{\mathcal{C}_{A}}\left(\hat{Z}_{A}^{1}, \hat{Z}_{A}^{N+1}\right)$ as the composite

$$
\hat{Z}_{A}^{1} \stackrel{\varphi_{1}}{\rightarrow} \hat{Z}_{A}^{2} \stackrel{\varphi_{2}}{\rightarrow} \cdots \stackrel{\varphi_{N+1}}{\rightarrow} \hat{Z}_{A}^{N+1} .
$$

Similarly we can take the generator $\varpi^{\prime}=\varphi_{1}^{\prime} \circ \cdots \varphi_{N}^{\prime}$ in $\operatorname{Hom}_{\mathcal{C}_{A}}\left(\hat{Z}_{A}^{N+1}, \hat{Z}_{A}^{1}\right)$. Then we have the following direct corollary to the above lemma.

Corollary 3.6. Keep the assumption and notations. Then

$$
\varpi^{\prime} \circ \varpi=t^{N(I, \lambda)} \mathbf{i d}_{\hat{Z}_{A}^{i}} \quad \text { and } \varpi \circ \varpi^{\prime}=t^{N(I, \lambda)} \mathbf{i d}_{\hat{Z}_{A}^{i+1}}
$$

where $N(I, \lambda)=\#\left\{\alpha \in R^{+} \backslash R_{I}^{+} \mid\left\langle\lambda+\rho, \alpha^{\vee}\right\rangle \neq 0 \bmod (p)\right\}$.

Lemma 3.7. (1) Let $\lambda, \mu \in X$. If $\operatorname{Ext}_{\mathcal{C}_{A}}^{1}\left(\hat{Z}_{A}(\lambda), \hat{Z}_{A}(\mu)\right) \neq 0$, then $\mu+\mathbb{Z} I \geq$ $\lambda+\mathbb{Z} I$.

(2) Assume that a module $M$ in category $\mathcal{C}_{A}$ has a $\hat{\mathcal{Z}}$-filtration. Then we can find a $\hat{\mathcal{Z}}$-filtration of $M$ :

$$
M=M_{0} \supset M_{1} \supset M_{2} \supset \cdots \supset M_{r} \supset M_{r+1}=0
$$

with $M_{i} / M_{i+1} \cong \hat{Z}_{A}\left(\lambda_{i}\right), \lambda_{i} \in X, i=0,1, \cdots, r$ satisfying that $\lambda_{i}+\mathbb{Z} I \geq$ $\lambda_{j}+\mathbb{Z} I$ implies $i \leq j$.

Proof. In analogy of [1, Lemma 2.14], we consider the exact sequence in the category $\mathcal{C}_{A}$ :

$$
0 \rightarrow \hat{Z}_{A}(\mu) \rightarrow N \rightarrow \hat{Z}_{A}(\lambda) \rightarrow 0
$$

Let $v \in N_{\lambda}$ be an inverse image of the standard generator $v_{0}=1 \otimes 1$ of $\hat{Z}_{A}(\lambda)$. If $x_{\alpha} v=0$ for all $\alpha \in R^{+}$, then there is a homomorphism $\hat{Z}_{A}(\lambda) \rightarrow N$ maps $v_{0}$ to $v$, splitting the above exact sequence. So, if the above exact sequence does not split, there must be $\alpha>0$ with $x_{\alpha} v \neq 0$. We get that $\lambda+\alpha$ is a weight of $M$, thereby is a weight of $\hat{Z}_{A}(\mu)$ because of the exact sequence. The first statement is proved. The second is a consequence of (1), by induction on the length of the filtration.

Remark 3.8. In Lemma 3.7(1), if we replace $\hat{Z}_{A}(\mu)$ and $\hat{Z}_{A}(\lambda)$ with $\hat{Z}_{A}^{w^{I}}\left(\mu^{w^{I}}\right)$ and $\hat{Z}_{A}^{w^{I}}\left(\lambda^{w^{I}}\right)$ respectively, we can get the similar result. In (2), if we replace $\hat{Z}$-filtration with $\hat{\mathcal{Z}}^{w^{I}}$-filtration, we can find that the $\hat{\mathcal{Z}}^{w^{I}}$-filtration of $M$ has the properties: if $\lambda_{i}+\mathbb{Z} I \geq \lambda_{j}+\mathbb{Z} I$, then $i \geq j$.

\section{Projective modues and $\hat{\mathcal{Z}}_{Q}-\hat{\mathcal{Z}}_{Q}^{w^{I}}$-filtrations}

The following theorem shows that the inverse of the statement in Proposition 2.4 concerning $\mathcal{Z}_{Q}$-filtrations is true.

Theorem 4.1. Maintain the assumption as in Proposition 2.4. Then $M$ is projective in the category $\mathcal{C}$ if and only if $M$ has both $\hat{\mathcal{Z}}_{Q^{-}}$and $\hat{\mathcal{Z}}_{Q}^{w^{I}}$-filtrations.

In order to prove the theorem, we need some knowledge about support varieties and rank varieties (one can refer to the definitions [5] [6] 7] and [10]). 
4.1. Support varieties and rank varieties. Given a vector space $V$ over $k$ let $V^{(-1)}$ denote the $k$-space with underlying abelian group $V$ and with scalars $\lambda \in k$ acting through multiplication by $\lambda^{p}$. Let $\|\mathfrak{g}\|$ denote the affine algebraic variety associated to the commutative Noetherian $k$-algebra $\bigoplus_{i \geq 0} \operatorname{Ext}_{U_{0}(\mathfrak{g})}^{2 i}(k, k)^{(-1)}$.

Definition 4.2. ([5, 6.1]) Let $M$ be a finite dimension $U_{\chi}(\mathfrak{g})$-module. The support variety $\|\mathfrak{g}\|_{M}$ of $M$ is the closed subvariety of $\|\mathfrak{g}\|$ given as the support of the $\operatorname{Ext}_{U_{0}(\mathfrak{g})}^{*}(k, k)^{(-1)}$-module $\operatorname{Ext}_{U_{\chi}(\mathfrak{g})}^{*}(M, M)^{(-1)}$.

There is a natural finite morphism $\Phi:\|\mathfrak{g}\| \rightarrow \mathfrak{g}$ of affine varieties (cf. [6, §2.1]), defined by using the $k$-algebra homomorphism

$$
S\left(\mathfrak{g}^{*}\right) \rightarrow \bigoplus_{i \geq 0} \operatorname{Ext}_{U_{0}(\mathfrak{g})}^{2 i}(k, k)^{(-1)}
$$

induced from the natural (Hochschild) map $\mathfrak{g}^{*} \rightarrow \operatorname{Ext}_{U_{0}(\mathfrak{g})}^{2}(k, k)^{(-1)}$. By Jantzen's theorem (cf. [13, Satz 2.14]), $\Phi(\|\mathfrak{g}\|)$ identifies with the closed subvariety $\mathcal{N}_{p}(\mathfrak{g})$ of $\mathfrak{g}$ where $\mathcal{N}_{p}(\mathfrak{g})=\left\{x \in \mathfrak{g} \mid x^{[p]}=0\right\}$. From the morphism $\Phi:\|\mathfrak{g}\| \rightarrow \mathcal{N}_{p}(\mathfrak{g})$, one can get a Zariski closed conical subset $\Phi\left(\|\mathfrak{g}\|_{M}\right)$ in $\mathcal{N}_{p}(\mathfrak{g})$, which can be identified with $\{0\} \bigcup\left\{x \in \mathcal{N}_{p}(\mathfrak{g})|M|_{k x}\right.$ is not a free $k x$-module $\}$ (cf. [5] and [7]).

Lemma 4.3. (cf. [5. Prop 6.2]) $M$ is a projective $U_{\chi}(\mathfrak{g})$-module if and only if $\Phi\left(\|\mathfrak{g}\|_{M}\right)=0$.

Now let us prove Theorem 4.1 .

Proof. $(\Longrightarrow)$ It is what Proposition 2.4 says.

$(\Longleftarrow)$ Suppose $M \in \mathcal{C}$ has a $\hat{\mathcal{Z}}_{Q}$-filtration and $\hat{\mathcal{Z}}_{Q}^{w^{I}}$-filtration. Observe that $M$ is projective in $\mathcal{C}$ if only if $\mathcal{F}(M)$ is a projective $U_{\chi}(\mathfrak{g})$-module. By Lemma 4.3. we only need to prove that $\Phi\left(\|\mathfrak{g}\|_{\mathcal{F}(M)}\right)=0$. Owing to [5. Prop 7.1], we know $\Phi\left(\|\mathfrak{g}\|_{\mathcal{F}(M) \otimes \mathcal{F}(M)}\right)=\Phi\left(\|\mathfrak{g}\|_{\mathcal{F}(M)}\right) \cap \Phi\left(\|\mathfrak{g}\|_{\mathcal{F}(M)}\right)$. Hence we only need consider $\Phi\left(\|\mathfrak{g}\|_{\mathcal{F}(M) \otimes \mathcal{F}(M)}\right)$.

As $M$ has a $\hat{\mathcal{Z}}_{Q}$-filtration and a $\hat{\mathcal{Z}}_{Q}^{w^{I}}$-filtration, $M \otimes M$ has a filtration where each sub-quotient in the filtration is isomorphic to $\hat{\mathcal{Z}}\left(Q, \lambda_{i}\right) \otimes M$ which admits another filtration with quotients of sub-quotients isomorphic to $\hat{\mathcal{Z}}\left(Q, \lambda_{i}\right) \otimes \hat{\mathcal{Z}}^{w^{I}}\left(Q, \lambda_{j}\right)$ for some $\lambda_{i}, \lambda_{j} \in X$. Hence, $\Phi\left(\|\mathfrak{g}\|_{\mathcal{F}(M) \otimes \mathcal{F}(M)}\right) \subset \bigcup_{i, j} \Phi\left(\|\mathfrak{g}\|_{\mathcal{Z}\left(Q, \lambda_{i}\right) \otimes \mathcal{Z}^{w^{I}}\left(Q, \lambda_{j}\right)}\right)$ (cf. [5] page 1085]). Associated with each component in the union, we have

$$
\Phi\left(\|\mathfrak{g}\|_{\mathcal{Z}\left(Q, \lambda_{i}\right) \otimes \mathcal{Z}^{w^{I}}\left(Q, \lambda_{j}\right)}\right)=\Phi\left(\|\mathfrak{g}\|_{\mathcal{Z}\left(Q, \lambda_{i}\right)}\right) \cap \Phi\left(\|\mathfrak{g}\|_{\mathcal{Z}^{w^{I}}\left(Q, \lambda_{j}\right)}\right) .
$$

By the same arguments in [5, Remark 7.5], we have $\Phi\left(\|\mathfrak{g}\|_{\mathcal{Z}\left(Q, \lambda_{j}\right)}\right) \subset \mathfrak{p}$ and $\Phi\left(\|\mathfrak{g}\|_{\mathcal{Z}^{w^{I}}\left(Q, \lambda_{j}\right)}\right) \subset w^{I}(\mathfrak{p})=\mathfrak{p}^{\prime}$. Thus,

$$
\Phi\left(\|\mathfrak{g}\|_{\mathcal{Z}\left(Q, \lambda_{i}\right) \otimes \mathcal{Z}^{w^{I}}\left(Q, \lambda_{j}\right)}\right)=\Phi\left(\|\mathfrak{g}\|_{\mathcal{Z}\left(Q, \lambda_{i}\right)}\right) \cap \Phi\left(\|\mathfrak{g}\|_{\mathcal{Z}^{w^{I}}\left(Q, \lambda_{j}\right)}\right) \subseteq \mathfrak{p} \cap \mathfrak{p}^{\prime}=\mathfrak{g}_{I} .
$$

On the other hand, both $\mathcal{Z}\left(Q, \lambda_{i}\right)$ and $\mathcal{Z}^{w^{I}}\left(Q, \lambda_{j}\right)$ are projective as $U_{\chi}\left(\mathfrak{g}_{I}\right)$ module, thereby both $\Phi\left(\|\mathfrak{g}\|_{\mathcal{Z}\left(Q, \lambda_{i}\right)}\right)$ and $\Phi\left(\|\mathfrak{g}\|_{\mathcal{Z}^{w^{I}}\left(Q, \lambda_{j}\right)}\right)$ intersect $\mathfrak{g}$ at 0 , owing to Lemma 4.3. Hence, $\Phi\left(\|\mathfrak{g}\|_{\mathcal{Z}\left(Q, \lambda_{i}\right)}\right) \cap \Phi\left(\|\mathfrak{g}\|_{\mathcal{Z}^{w^{I}}\left(Q, \lambda_{j}\right)}\right)=0$. Thus, we have proved that $\Phi(\| \mathfrak{g}||)_{M}=0$. Hence, $M$ is projective. The proof is completed.

We immediately have the following corollary. 
Corollary 4.4. If $M$ is a projective module in $\mathcal{C}$, then ${ }^{\tau} M$ must be projective in ${ }^{\tau} \mathcal{C}$, where ${ }^{\tau} \mathcal{C}$ is the $X / \mathbb{Z} I$-graded module category of $U_{-\chi}(\mathfrak{g})$. In particular, in this case ${ }^{\tau} M$ admits a filtration with filtration quotient factors ${ }^{\tau} \hat{Z}(\lambda)$ for some $\lambda \in X$.

Remark 4.5. By Remarks 2.5(2) and2.7 the $\mathcal{C}_{A}$-version of Corollary 4.4 is also true. This is to say, if $\hat{Q}$ is projective in $\mathcal{C}_{A}$, then ${ }^{\tau} \hat{Q}$ admits a filtration with filtration quotient factors like ${ }^{\tau} \hat{Z}_{A}(\lambda)$.

Proposition 4.6. (Compare with [1, Proposition 4.11]) Let $\chi$ be of standard Levi form associated with a proper subset $I$ of simple roots. Then

$$
\operatorname{Ext}_{\mathcal{C}}^{n}\left(\hat{\mathcal{Z}}(Q, \lambda), \hat{\mathcal{Z}}^{w^{I}}\left(Q, \mu^{w^{I}}\right)\right)=0
$$

for all $\lambda, \mu \in X$ and $n>0$.

Proof. Since $\hat{\mathcal{Z}}(Q, \lambda)$ is a $U_{\chi}(\mathfrak{g})$-module, we know that its dual $\hat{\mathcal{Z}}(Q, \lambda)^{*}$ is a $U_{-\chi}(\mathfrak{g})$-module. Hence, we can get by [5, Proposition 5.1]

$$
\operatorname{Ext}_{\mathcal{C}}^{n}\left(\hat{\mathcal{Z}}(Q, \lambda), \hat{\mathcal{Z}}^{w^{I}}\left(Q, \mu^{w^{I}}\right)\right) \simeq \mathrm{H}^{n}\left(U_{0}(\mathfrak{g}), \hat{\mathcal{Z}}(Q, \lambda)^{*} \otimes \hat{\mathcal{Z}}^{w^{I}}\left(Q, \mu^{w^{I}}\right)\right)
$$

Noticing the fact $\Phi\left(\|\mathfrak{g}\|_{\mathcal{Z}(Q, \lambda)}\right)=\Phi\left(\|\mathfrak{g}\|_{\mathcal{Z}\left(Q, \lambda_{i}\right)^{*}}\right)$, we can get that by [5. Proposition $6.2]$

$$
\Phi\left(\|\mathfrak{g}\|_{\mathcal{Z}(Q, \lambda) * \otimes \mathcal{Z}^{w^{I}}\left(Q, \mu^{w^{I}}\right)}\right) \subset \Phi\left(\|\mathfrak{g}\|_{\mathcal{Z}\left(Q, \lambda_{i}\right)}\right) \cap \Phi\left(\|\mathfrak{g}\|_{\mathcal{Z}^{w^{I}}\left(Q, \mu^{w^{I}}\right)}\right)=0,
$$

the reason of which is the same as in the proof of Theorem 2.4. Then by Lemma 4.3. we can know that $\hat{\mathcal{Z}}(Q, \lambda)^{*} \otimes \hat{\mathcal{Z}}^{w^{I}}\left(Q, \mu^{w^{I}}\right)$ is a projective $U_{0}(\mathfrak{g})$-module, as well as an injective module because $U_{0}(\mathfrak{g})$ is a Frobenius algebra. Thus we have $\mathrm{H}^{n}\left(U_{0}(\mathfrak{g}), \hat{\mathcal{Z}}(Q, \lambda)^{*} \otimes \hat{\mathcal{Z}}^{w^{I}}\left(Q, \mu^{w^{I}}\right)\right)=0$ and the lemma is proved.

\section{Andersen-Kaneda filtrations and sum formulas}

Maintain the notations as the previous sections. Especially, we set $A=k[t]_{(t)}$ the localization of the polynomial ring $k[t]$ in one variable at the maximal ideal generated by $t$, we will denote by $\tilde{A}$ the fraction field of $k[t]$.

5.1. Andersen-Kaneda filtrations. In the situation of [2, §3], Andersen and Kaneda constructed a filtration of the vector space $F_{\lambda}(Q)=\operatorname{Hom}_{G_{1} T}\left(Z(\lambda)^{\tau}, Q\right)$ for each projective $G_{1} T$-module $Q$, where $\tau$ denotes the contra-variant dual [2,, 1.6$]$. We find that there exist the similar filtration in the representation theory of modular Lie algebra of $p$-character $\chi$ when $\chi$ has standard Levi form. We will define this filtration in our situation and study its properties analogous to that in [2, $\S 3]$.

Let $\hat{Q}$ be the projective module in category $\mathcal{C}_{A}$. As stated in Remark 2.5, there is a unique projective module $\hat{Q}_{k}$ in category $\mathcal{C}$ with $\hat{Q}_{k} \otimes_{A} k \simeq \hat{Q}$. Recall that $\operatorname{Hom}_{\mathcal{C}_{A}}\left(\hat{Z}_{A}^{w^{I}}\left(\lambda^{w^{I}}\right), \hat{Z}_{A}(\lambda)\right) \cong A($ Lemma $3.2(2))$. And there is a unique generator $c:=$ $\varpi^{\prime}$ as appearing before Corollary [3.6, up to units in $A$, in $\operatorname{Hom}_{\mathcal{C}_{A}}\left(\hat{Z}_{A}^{w^{I}}\left(\lambda^{w^{I}}\right), \hat{Z}_{A}(\lambda)\right)$.

Define

$$
\begin{gathered}
F_{A}^{\lambda}(\hat{Q})=\operatorname{Hom}_{\mathcal{C}_{A}}\left(\hat{Z}_{A}^{w^{I}}\left(\lambda^{w^{I}}\right), \hat{Q}\right), \\
E_{A}^{\lambda}(\hat{Q})=\operatorname{Hom}_{\mathcal{C}_{A}}\left(\hat{Q}, \hat{Z}_{A}(\lambda)\right) .
\end{gathered}
$$

By Lemmas 2.8, Corollary 4.4 and Remark 2.5, it's not hard to see that

$$
F_{A}^{\lambda}(\hat{Q}) \text { and } E_{A}^{\lambda}(\hat{Q}) \text { are both } A \text {-free module with the same rank, say } n_{\lambda} \text {. }
$$


Set $F_{k}^{\lambda}\left(\hat{Q}_{k}\right)=\operatorname{Hom}_{\mathcal{C}}\left(\hat{Z}_{\chi}^{w^{I}}\left(\lambda^{w^{I}}\right), \hat{Q}_{k}\right)$ and $E_{k}^{\lambda}\left(\hat{Q}_{k}\right)=\operatorname{Hom}_{\mathcal{C}_{k}}\left(\hat{Q}_{k}, \hat{Z}_{\chi}(\lambda)\right)$. The projectiveness of $\hat{Q}$ implies by [1, Proposition 3.3] that

$$
F_{k}^{\lambda}\left(\hat{Q}_{k}\right) \simeq F_{A}^{\lambda}(\hat{Q}) \otimes_{A} k,
$$

and $E_{k}^{\lambda}\left(\hat{Q}_{k}\right) \simeq E_{A}^{\lambda}(\hat{Q}) \otimes_{A} k$.

Similar to that in [2, $\S 3]$, define a filtration $\left\{F_{A}^{\lambda}(\hat{Q})^{(j)}\right\}_{j \geq 0}$ by setting

$$
F_{A}^{\lambda}(\hat{Q})^{(j)}=\left\{\varphi \in F_{A}^{\lambda}(\hat{Q}) \mid \psi \circ \varphi \in A t^{j} c, \psi \in E_{A}^{\lambda}(\hat{Q})\right\}
$$

and let $F_{k}^{\lambda}\left(\hat{Q}_{k}\right)^{(j)}$ be the image of $F_{A}^{\lambda}(\hat{Q})^{(j)}$ in $F_{k}^{\lambda}\left(\hat{Q}_{k}\right)$, then

$$
F_{k}^{\lambda}\left(\hat{Q}_{k}\right)^{(j)} \simeq\left(F_{A}^{\lambda}(\hat{Q})^{(j)}+t F_{A}^{\lambda}(\hat{Q})\right) / t F_{A}^{\lambda}(\hat{Q}) \simeq F_{A}^{\lambda}(\hat{Q})^{(j)} / t F_{A}^{\lambda}(\hat{Q})^{(j-1)} .
$$

We call such a filtration of the projective module $Q$ an Andersen-Kaneda filtration (or AK filtration).

Thanks to Lemma 3.3(2), we have the pairing

$$
a_{\lambda}: F_{A}^{\lambda}(\hat{Q}) \times E_{A}^{\lambda}(\hat{Q}) \longrightarrow A
$$

given by $\psi \circ \varphi=a_{\lambda}(\varphi, \psi) c, \psi \in E_{A}^{\lambda}(\hat{Q}), \varphi \in F_{A}^{\lambda}(\hat{Q})$. When tensored with $\tilde{A}$ we have a bilinear $\tilde{A}$-form $(-,-)$ arising from this pairing. This $\tilde{A}$-bilinear form is by definition non-degenerate. Furthermore, there is with the pairing, an $A$-homomorphism

$$
\theta_{\lambda}: F_{A}^{\lambda}(\hat{Q}) \longrightarrow E_{A}^{\lambda}(\hat{Q})^{\vee}=\operatorname{Hom}_{A}\left(E_{A}^{\lambda}(\hat{Q}), A\right)
$$

defined by

$$
\theta_{\lambda}(\varphi): \psi \mapsto a_{\lambda}(\varphi, \psi)
$$

From the non-degeneracy of the $\tilde{A}$-bilinear form, $\theta_{\lambda}$ is an $\tilde{A}$-isomorphism, when tensorred with $\tilde{A}$. There are some basic facts with $\theta$ as follows.

Lemma 5.1. There exist bases in $F_{A}^{\lambda}(\hat{Q})$ and $E_{A}^{\lambda}(\hat{Q}):\left\{f_{1}, f_{2}, \cdots f_{n_{\lambda}}\right\},\left\{e_{1}, e_{2}, \cdots e_{n_{\lambda}}\right\}$ respectively, together with a sequence of positive integers $\left\{m_{\lambda}(1), m_{\lambda}(2), \cdots, m_{\lambda}\left(n_{\lambda}\right)\right\}$ such that

$$
\theta_{\lambda}\left(f_{i}\right)=t^{m_{\lambda}(i)} e_{i}, i=1,2, \cdots, n_{\lambda}
$$

Moreover,

$$
\sum_{j \geq 1} \operatorname{dim} F_{k}^{\lambda}\left(\hat{Q}_{k}\right)^{(j)}=\nu_{t} \operatorname{det}\left(\theta_{\lambda}\right)=\sum_{i=1}^{n_{\lambda}} m_{\lambda}(i)
$$

Proof. Note that both $F_{A}^{\lambda}(\hat{Q})$ and $E_{A}^{\lambda}(\hat{Q})$ are $A$-free of rank $n_{\lambda}$, and $\theta_{\lambda}$ is an $\tilde{A}$-isomorphism. For a given basis $\left\{e_{1}, \cdots, e_{n_{\lambda}}\right\}$ in $E_{A}^{\lambda}(\hat{Q})$, there must exist a basis $\left\{f_{1}, \cdots, f_{n_{\lambda}}\right\}$ in $F_{A}^{\lambda}(\hat{Q})$ such that

$$
\theta_{\lambda}\left(f_{i}\right)=t^{m_{\lambda}(i)} e_{i}, i=1,2, \cdots, n_{\lambda} .
$$

As to the second formula, it follows from the standard arguments as in [12, II §8.18]. 
5.2. Connection with Jantzen filtrations. Recall there are subspace filtrations on Weyl modules in representations of algebraic groups (cf. [12]). It's usually called Jatzen's filtration. The idea can be adopted to the Lie algebra case when $\chi$ is of standard Levi forms. Recall there are up to units in $A$, unique generators $c:=\varpi^{\prime}$ and $c^{\prime}:=\varpi$ respectively in $\operatorname{Hom}_{A}\left(\hat{Z}_{A}^{w^{I}}\left(\lambda^{w^{I}}\right), \hat{Z}_{A}(\lambda)\right)$ and in $\operatorname{Hom}_{A}\left(\hat{Z}_{A}(\lambda), \hat{Z}_{A}^{w^{I}}\left(\lambda^{w^{I}}\right)\right)$ (see the first subsection). According to Corollary 3.6, we have

$$
c \circ c^{\prime}=t^{N(I, \lambda)} \operatorname{id}_{\hat{Z}_{A}^{w I}\left(\lambda^{w^{I}}\right)} \cdot
$$

In our case, the Jantzen filtration on $\hat{Z}_{A}^{w^{I}}\left(\lambda^{w^{I}}\right)$ and $Z_{A}(\lambda)$ can be defined some sequences of their vector subspaces respectively defined via

$$
\begin{aligned}
& \hat{Z}_{A}^{w^{I}}\left(\lambda^{w^{I}}\right)^{(j)}=\left\{v \in \hat{Z}_{A}^{w^{I}}\left(\lambda^{w^{I}}\right) \mid c v \in t^{j} \hat{Z}_{A}(\lambda)\right\} \\
& \hat{Z}_{A}(\lambda)^{(j)}=\left\{v^{\prime} \in \hat{Z}_{A}(\lambda) \mid c^{\prime} v^{\prime} \in t^{j} \hat{Z}_{A}^{w^{I}}\left(\lambda^{w^{I}}\right)\right\} .
\end{aligned}
$$

(cf. [11, 3.8]).

As argument in the proof of Lemma 3.2 and Remark 3.5, it's not hard to see there exist bases $\left\{v_{1}, v_{2}, \cdots, v_{n}\right\}$ of $\hat{Z}_{A}^{w^{I}}\left(\lambda^{w^{I}}\right)$ and bases $\left\{v_{1}^{\prime}, v_{2}^{\prime}, \cdots, v_{n}^{\prime}\right\}$ of $\hat{Z}_{A}(\lambda)$ and integers $a_{1}, a_{2}, \cdots, a_{n} \in \mathbb{N}$ such that

$$
c v_{i}=t^{a_{i}} v_{i}^{\prime}, \quad \quad \quad c^{\prime} v_{i}^{\prime}=t^{N(I, \lambda)-a_{i}} v_{i} .
$$

We denote by $\hat{Z}_{\chi}^{w^{I}}\left(\lambda^{w^{I}}\right)^{(j)}$ (resp. $\left.\hat{Z}_{\chi}(\lambda)\right)$ the image of $\hat{Z}_{A}^{w^{I}}\left(\lambda^{w^{I}}\right)^{(j)}$ in $Z_{\chi}^{w^{I}}\left(\lambda^{w^{I}}\right)$ (resp. the image of $\hat{Z}_{A}(\lambda)^{(j)}$ in $\hat{Z}_{\chi}(\lambda)$ ), then we have (set $\bar{v}_{i}=v_{i} \otimes 1, \bar{v}_{i}{ }^{\prime}=v_{i}^{\prime} \otimes 1$ )

$$
\begin{gathered}
\hat{Z}_{\chi}^{w^{I}}\left(\lambda^{w^{I}}\right)^{(j)}=\sum_{\substack{i \\
a_{i} \geq j}} k \bar{v}_{i}, \\
\hat{Z}_{\chi}(\lambda)^{(j)}=\sum_{N(I, \lambda)-a_{i} \geq j}^{i} k \bar{v}_{j}^{\prime}
\end{gathered}
$$

Observing that $\bar{v}_{i}$ and $\bar{v}_{i}^{\prime}$ have the same weight we deduce from (5.8)

$$
\operatorname{ch} \hat{Z}_{\chi}^{w^{I}}\left(\lambda^{w^{I}}\right)^{(j)}+\operatorname{ch} \hat{Z}_{\chi}(\lambda)^{(N(I, \lambda)-j+1)}=\operatorname{ch} \hat{Z}_{\chi}(\lambda)
$$

Let's return to the AK filtration. With the above arguments, we know $\hat{Q}_{A}$ has a $\hat{\mathcal{Z}}_{A}^{w^{I}}$-filtration,

$$
\hat{Q}_{A}=\hat{Q}_{A}^{(0)} \supset \hat{Q}_{A}^{(1)} \supset \cdots \supset \hat{Q}_{A}^{(r)}=0
$$

with

$$
\hat{Q}_{A}^{(i-1)} / \hat{Q}_{A}^{(i)} \simeq \hat{Z}_{A}^{w^{I}}\left(\lambda_{i}^{w^{I}}\right)^{n_{i}}, n_{i}=\left(\hat{Q}_{A}: \hat{Z}_{A}\left(\lambda_{i}\right)\right)
$$

satisfying as in Remark 3.8 that

$$
\text { if } \lambda_{i}+\mathbb{Z} I \leq \lambda_{j}+\mathbb{Z} I \text {, then } i \geq j
$$

for $i=1,2, \cdots, r$.

We can get an exact sequence

$$
\begin{aligned}
0 \rightarrow \operatorname{Hom}_{\mathcal{C}_{A}}\left(\hat{Z}_{A}^{w^{I}}\left(\lambda_{i}^{w^{I}}\right), \hat{Q}_{A}^{(i-1)}\right) & \rightarrow \operatorname{Hom}_{\mathcal{C}_{A}}\left(\hat{Z}_{A}^{w^{I}}\left(\lambda_{i}^{w^{I}}\right), \hat{Z}_{A}^{w^{I}}\left(\lambda_{i}^{w^{I}}\right)^{n_{i}}\right) \\
& \rightarrow \operatorname{Ext}_{\mathcal{C}_{A}}^{1}\left(\hat{Z}_{A}^{w^{I}}\left(\lambda_{i}^{w^{I}}\right), \hat{Q}_{A}^{(i)}\right) \rightarrow 0 .
\end{aligned}
$$


which follows from the exact sequence:

$$
0 \rightarrow \hat{Q}_{A}^{(i)} \rightarrow \hat{Q}_{A}^{(i-1)} \rightarrow \hat{Z}_{A}^{w^{I}}\left(\lambda_{i}^{w^{I}}\right)^{n_{i}} \rightarrow 0
$$

operated by the functor $\operatorname{Hom}_{\mathcal{C}_{A}}\left(\hat{Z}_{A}^{w^{I}}\left(\lambda_{i}^{w^{I}}\right),-\right)$, as well as the fact that

$$
\operatorname{Hom}_{\mathcal{C}_{A}}\left(\hat{Z}_{A}^{w^{I}}\left(\lambda_{i}^{w^{I}}\right), \hat{Q}_{A}^{(i)}\right)=0 .
$$

Furthermore, the inclusion $\hat{Q}_{A}^{(i-1)} \hookrightarrow \hat{Q}_{A}$ induces an isomorphism

$$
\operatorname{Hom}_{\mathcal{C}_{A}}\left(\hat{Z}_{A}^{w^{I}}\left(\lambda_{i}^{w^{I}}\right), \hat{Q}_{A}^{(i-1)}\right) \simeq \operatorname{Hom}_{\mathcal{C}_{A}}\left(\hat{Z}_{A}^{w^{I}}\left(\lambda_{i}^{w^{I}}\right), \hat{Q}_{A}\right)=F_{A}^{\lambda_{i}}(\hat{Q}),
$$

because $\hat{Q}_{A} / \hat{Q}_{A}^{(i-1)}$ is filtered by $\hat{Z}_{A}^{w^{I}}\left(\lambda_{j}^{w^{I}}\right), j>i$.

Set $\widehat{F_{A}^{\lambda}(\hat{Q})}=\operatorname{Hom}_{\mathcal{C}_{A}}\left(\hat{Z}_{A}(\lambda), \hat{Q}_{A}\right)$. Then it's not hard to see the following fact by summing up the above arguments (the detailed proof may be referred to [2, 3.5]).

Lemma 5.2. There exist A-bases $\left\{\psi_{1}, \psi_{2}, \cdots, \psi_{r}\right\}$ of $E_{A}^{\lambda}(\hat{Q})$ and $\left\{\psi_{1}^{\prime}, \psi_{2}^{\prime}, \cdots, \psi_{r}^{\prime}\right\}$, of $\widetilde{F_{A}^{\lambda}(\hat{Q})}$ such that

$$
\psi_{i} \circ \psi_{j}^{\prime}=\delta_{i j} t^{N(I, \lambda)} i d_{\hat{Z}_{A}(\lambda)}, i, j=1,2, \cdots, r .
$$

Recall for $\varphi \in F_{A}^{\lambda}(\hat{Q})$ and $\psi \in E_{A}^{\lambda}(\hat{Q})$, we have $\psi \circ \varphi=a_{\lambda}(\varphi, \psi) c$. Hence $c \circ c^{\prime}=t^{N(I, \lambda)} \mathbf{i d}_{\hat{Z}_{A}^{N+1}}$ implies implies

$$
\psi \circ \varphi \circ c^{\prime}=a_{\lambda}(\varphi, \psi) t^{N(I, \lambda)} \mathbf{i d}_{\hat{Z}_{A}(\lambda)}
$$

With the bases from the above lemma, we may write $\varphi \circ c^{\prime}=\sum_{s=1}^{r} b_{s} \psi_{s}^{\prime}$ for some $b_{s} \in A$. Furthermore, $b_{s}=a_{\lambda}\left(\varphi, \psi_{s}\right)$ for all $s$. Hence $\varphi \circ c^{\prime} \in t^{j} F_{A}^{\lambda}(\hat{Q})$ if and only if $t^{j} \mid a_{\lambda}\left(\varphi, \psi_{s}\right)$ for all $s$, i.e., if and only if $\varphi \in F_{A}^{\lambda}(\hat{Q})^{(j)}$.

Thus, we have a corollary to the above lemma.

Lemma 5.3. For each $j \in \mathbb{N}$ we have

$$
F_{A}^{\lambda}(\hat{Q})^{(j)}=\left\{\psi \in F_{A}^{\lambda}(\hat{Q}) \mid \varphi \circ c^{\prime} \in t^{j} \widetilde{F_{A}^{\lambda}(\hat{Q})}\right\} .
$$

5.3. Sum formulas over $\mathcal{C}$. Now we are in the position to present the main results about AK filtrations and the related sum formulas.

Lemma 5.4. $\operatorname{Ker} \bar{\varphi}_{i} \cong \operatorname{Coker} \bar{\varphi}_{i}$, where $\bar{\varphi}_{i}$ is an extension of $\varphi_{i}$ by change of rings $-\otimes_{A} k$, and $\varphi_{i}$ is defined as in Lemma 3.4.

ProOF. It follows from [11, Lemma 3.5].

Theorem 5.5. Let $\nu \in X$. Then

$$
\sum_{j \geq 1} \operatorname{dim} F_{k}^{\lambda}\left(\hat{Q}_{\chi}(\nu)\right)^{(j)}=\sum_{j \geq 1}\left[\hat{Z}_{\chi}(\lambda)^{(j)}: \hat{L}_{\chi}(\nu)\right]
$$

Proof. Let $\hat{Q}=\hat{Q}_{\chi}(\nu) \in \mathcal{C}, \hat{Q}_{A}=\hat{Q}_{A}(\nu) \in \mathcal{C}_{\mathcal{A}}$. Denote by $\phi_{i}: \operatorname{Hom}_{\mathcal{C}_{A}}\left(\hat{Z}_{A}^{i+1}, \hat{Q}_{A}\right) \rightarrow$ $\operatorname{Hom}_{\mathcal{C}_{A}}\left(\hat{Z}_{A}^{i}, \hat{Q}_{A}\right)$ which is induced by $\varphi_{i}$. Here $\varphi_{i}$ is defined as in Lemma 3.4. Let $\phi=\phi_{1} \circ \cdots \circ \phi_{N}$. Then Lemma 5.3 says

$$
F_{A}^{\lambda}(\hat{Q})^{(j)}=\left\{\varphi \in F_{A}^{\lambda}(Q) \mid \phi(\varphi) \in t^{j} \widetilde{F_{A}^{\lambda}(Q)}\right\}
$$


Analogy of the arguments as in $\$ 5.1$ gives the following sum formula.

$$
\sum_{j \geq 1} \operatorname{dim} F_{k}^{\lambda}(\hat{Q})^{(j)}=\nu_{t}(\operatorname{det} \phi)=\sum_{i=1}^{N} \nu_{t}\left(\operatorname{det} \phi_{i}\right) .
$$

Set $C_{i}=\operatorname{Coker} \varphi_{i}$. Then by [11, $\left.\S 3.7\right]$, we have

$$
t C_{i}=0, \text { for all i. }
$$

Hence we also have

$$
t \operatorname{Ext}_{\mathcal{C}_{A}}^{1}\left(C_{i}, \hat{Q}_{A}\right)=0 \text {, for all i. }
$$

Notice that the homomorphism $\hat{Z}_{A}^{i-1} \rightarrow \hat{Z}_{A}^{i}$ is injective, we have the short exact sequence

$$
0 \rightarrow \hat{Z}_{A}^{i} \stackrel{\varphi_{i}}{\rightarrow} \hat{Z}_{A}^{i+1} \stackrel{\tilde{\varphi}_{i}}{\rightarrow} C_{i} \rightarrow 0 .
$$

Moreover we can get the exact sequence through the action by $\operatorname{Hom}_{\mathcal{C}_{\mathcal{A}}}\left(-, \hat{Q}_{A}\right)$

$$
0 \rightarrow \operatorname{Hom}_{\mathcal{C}_{\mathcal{A}}}\left(\hat{Z}_{A}^{i+1}, \hat{Q}_{A}\right) \stackrel{\phi_{i}}{\rightarrow} \operatorname{Hom}_{\mathcal{C}_{\mathcal{A}}}\left(\hat{Z}_{A}^{i}, \hat{Q}_{A}\right) \rightarrow \operatorname{Ker} \Phi_{i} \rightarrow 0
$$

where $\Phi_{i}: \operatorname{Ext}_{\mathcal{C}_{A}}^{1}\left(C_{i}, \hat{Q}_{A}\right) \rightarrow \operatorname{Ext}_{\mathcal{C}_{A}}^{1}\left(\hat{Z}_{A}^{i+1}, \hat{Q}_{A}\right)$. It is clear that $\operatorname{Ker} \Phi_{i}$ is the submodule of $\operatorname{Ext}_{\mathcal{C}_{A}}^{1}\left(C_{i}, \hat{Q}_{A}\right)$. Hence (5.16) implies

$$
\nu_{t}\left(\operatorname{det} \phi_{i}\right)=\operatorname{dim}_{k}\left(\operatorname{Ker} \Phi_{i} \otimes_{A} k\right)
$$

Set $\bar{\varphi}_{i}=\varphi_{i} \otimes_{A} k, \bar{C}_{i}=C_{i} \otimes_{A} k$. Then $\bar{C}_{i}=\operatorname{Coker} \bar{\varphi}_{i}$ and the injectivity of $\hat{Q}$ gives the exactness of the top row in the following diagram (note that $U_{\chi}(\mathfrak{g})$ is a Frobenius algebra, the projective $U_{\chi}(\mathfrak{g})$-modules is injective):

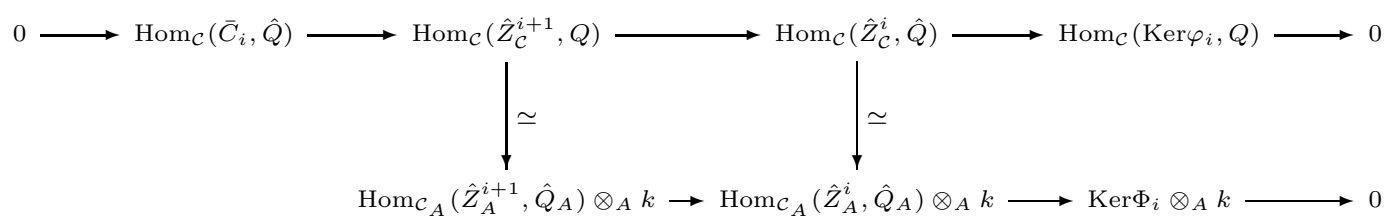

Here the bottom sequence is obtained by tensoring (5.18) with $k$ and the vertical isomorphism comes from the application of Proposition 2.4 and Lemma 3.3 along with Remarks 2.5(2), 2.7 and 4.5. The diagram shows that $\operatorname{Ker} \Phi_{i} \otimes_{A} k$ may be identified with $\operatorname{Hom}_{\mathcal{C}}\left(\operatorname{Ker} \bar{\varphi}_{i}, \hat{Q}\right)$, thereby this has the same dimension as $\operatorname{Hom}_{\mathcal{C}}\left(\bar{C}_{i}, \hat{Q}\right)$, owing to Lemma 5.4. By Lemma 2.6 and Remark 2.7, it can be seen that this dimension equals the multiplicity $\left[\bar{C}_{i}, \hat{L}_{\chi}(\mu)\right]$. Consider $\bar{C}_{i}=\operatorname{Ker} \bar{\varphi}_{i}$ (Lemma 5.4), which is decided by the formula in Remark 3.5. Hence, the arguments on (5.7)-(5.9) tells us

$$
\sum_{j \geq 1} \operatorname{ch} \hat{Z}_{\chi}(\lambda)^{(j)}=\sum_{i=1}^{N} \operatorname{ch} \bar{C}_{i}
$$


Combining (5.15), (5.19) and (5.20), we know

$$
\sum_{j \geq 1} \operatorname{dim} F_{k}^{\lambda}(\hat{Q})^{(j)}=\sum_{i=1}^{N}\left[\bar{C}_{i}: \hat{L}_{\chi}(\nu)\right]=\sum_{j \geq 1}\left[\hat{Z}_{\chi}(\lambda)^{(j)}: \hat{L}_{\chi}(\nu)\right] .
$$

The proof is completed.

The following result shows the connection between the dimension of individual terms between AK filtrations and Jantzen's filtrations.

Theorem 5.6. Let $\hat{Q} \in \mathcal{C}$ be projective. Then we have a formula $\operatorname{dim} F_{k}^{\lambda}(\hat{Q})^{(j)}=$ $\operatorname{dimHom}\left(\hat{Z}_{\chi}(\lambda)^{(j)}, \hat{Q}\right)$, for all $j \in \mathbb{N}$. Especially, for $\hat{Q}=\hat{Q}(\nu), \nu \in X, \operatorname{dim} F_{k}^{\lambda}(\hat{Q}(\nu))^{(j)}=$ $\left[\hat{Z}_{\chi}(\lambda)^{(j)}: \hat{L}_{\chi}(\nu)\right]$, for all $j$.

Proof. The second part is a direct implication of the first one. It's sufficient to prove the first one. For this, let us verify the statement below.

$$
\text { If } \bar{\varphi} \in F_{k}^{\lambda}(\hat{Q})^{(j)} \text {, then } \bar{\varphi}\left(\hat{Z}_{\chi}^{w^{I}}\left(\lambda^{w^{I}}\right)^{(N(I, \lambda)+1-j)}\right)=0 \text {. }
$$

We denote $\bar{\varphi} \in F_{k}^{\lambda}(\hat{Q})^{(j)}$ is the image of some $\varphi \in F_{A}^{\lambda}(\hat{Q})^{(j)}$ in $F_{k}^{\lambda}(\hat{Q})$. By Proposition 5.3 we have $\varphi \circ c^{\prime} \in t^{j} \widetilde{F_{A}^{\lambda}(\hat{Q})}$. On the other hand, by (5.7) we have

$$
\varphi \circ c^{\prime}\left(v_{i}^{\prime}\right)=t^{N(I, \lambda)-a_{i}} \varphi\left(v_{i}\right), i=1,2, \cdots n
$$

We conclude that

$$
\text { if } N(I, \lambda)-a_{i} \leq j \text { then } \varphi\left(v_{i}\right) \in t^{j-N(I, \lambda)+a_{i}} \hat{Q}_{A}
$$

By (5.8) we see that $\hat{Z}_{A}^{w^{I}}\left(\lambda^{w^{I}}\right)^{(N(I, \lambda)+1-j)}$ is spanned by $\bar{v}_{i}$ where $a_{i} \geq N(I, \lambda)+1-j$. Hence (5.22) implies that if $\bar{v}_{i} \in Z_{A}^{w^{I}}\left(\lambda^{w^{I}}\right)^{(N(I, \lambda)+1-j)}$, then $\varphi\left(v_{i}\right) \in t \hat{Q}_{A}$. That is to say that $\bar{\varphi}\left(\bar{v}_{i}\right)=0$, thereby (5.21) is proved.

According to (5.21), we see that

$$
F_{k}^{\lambda}(\hat{Q})^{j} \subseteq \operatorname{Hom}_{\mathcal{C}_{A}}\left(\hat{Z}_{\chi}^{w^{I}}\left(\lambda^{w^{I}}\right) / \hat{Z}_{\chi}^{w^{I}}\left(\lambda^{w^{I}}\right)^{(N(I, \lambda)+1-j)}, \hat{Q}\right)
$$

Since $\hat{Q}$ is injective, the dimension of this homomorphism space only depends on the character of $\hat{Z}_{\chi}^{w^{I}}\left(\lambda^{w^{I}}\right) / \hat{Z}_{\chi}^{w^{I}}\left(\lambda^{w^{I}}\right)^{(N(I, \lambda)+1-j)}$. Therefore by (5.9) we deduce

$$
\operatorname{dim} F_{k}^{\lambda}(\hat{Q})^{(j)} \leq \operatorname{dimHom}_{\mathcal{C}_{A}}\left(\hat{Z}_{\chi}(\lambda), \hat{Q}\right)^{(j)} \text { for all } \mathrm{j} \in \mathbb{N} .
$$

On the other hand, each finite-dimensional projective object in $\mathcal{C}$ is the direct sum of some $Q(\nu)$. Thus Theorem 5.5 make in force the equality true. The proof is completed.

Remark 5.7. For each projective module $\hat{Q} \in \mathcal{C}$ we have

(1) The length of the Jantzen filtration of $\hat{Z}_{\chi}(\lambda)$ is just $N(I, \lambda)$. So it follows from the above theorem that

$$
F_{k}^{\lambda}(\hat{Q})^{(N(I, \lambda)+1)}=0 \text { for all projective modules } \hat{Q} \in \mathcal{C} .
$$

(2) We have another version of the sum formula

$$
\begin{aligned}
\sum_{j \geq 1} \operatorname{dim} F_{k}^{\lambda}\left(\hat{Q}^{(j)}\right)= & \sum_{\alpha \in R^{+}(\lambda) \backslash R_{I}^{+}}\left(\sum_{i \geq 0}\left(\hat{Q}: \hat{Z}_{A}\left(\lambda-\left(i p+n_{\alpha}\right)\right)\right)-\right. \\
& \left.\sum_{i \geq 1}\left(\hat{Q}: \hat{Z}_{A}(\lambda-i p \alpha)\right)\right) .
\end{aligned}
$$


(3) $\operatorname{dim} F_{k}^{\lambda}(\hat{Q})_{0}=(\hat{Q}: \hat{Q}(\lambda))$ and $\operatorname{dim} F_{k}^{\lambda}(\hat{Q})_{N(I, \lambda)}=\left(\hat{Q}: \hat{Q}\left(\lambda^{\prime}\right)\right)$ where $\hat{L}\left(\lambda^{\prime}\right)=$ $\operatorname{Soc}\left(\hat{Z}_{\chi}(\lambda)\right)$.

\section{References}

[1] Andersen H. H., Jantzen J. C. and Soergel, W., Representations of quantum groups at a pth root of unity and of semisimple groups in charactertic p: independence of p., Asterisque 220 (1994).

[2] Andersen H. H. and Kaneda, M., Filtrations on $G_{1} T$-modules, Proc. Londen Math. Soc. 82 (2001), 614-646.

[3] Cline E., Parshall B. and Scott, L., On injective modules for infinitesimal algebraic groups I, J. London Math. Soc.(2) 31 (1985), 277-291

[4] Curtis C.W. and Reiner I., Methods of representation theory. With applications to finite groups and orders, Vol.I, A Wiley-Interscience Publication, New York, 1981.

[5] Friedlander E. M. and Parshall B., Modular representation theory of Lie algebras, Amer. J. Math. 110 (1988), 1055-1093.

[6] Friedlander E. M. and Parshall B., Geometry of p-unipotent Lie algebras, J. Algebra 109 (1987), 25-45

[7] Friedlander E. M. and Parshall B., Support varieties for restricted Lie algebra, Invent. math. 86 (1986),553-562

[8] Friedlander E. M. and Parshall B., Deformations of Lie algebra representations, Amer. J. Math. 112(1990), 375-395.

[9] Humphreys J.. Modular representations of classical Lie algebras and semisimple groups, J. Algebra 19 (1970), 51-79

[10] Jantzen J. C., Representations of Lie algebras in prime characteristic, in ; A. Broer (Ed. ), Representation Theories and Algebraic Geometry, Proceedings, Montreal, 1997, in: NATO ASI Series, Vol. C514, Kluwer, Dordrecht, 1998, pp. 185-235.

[11] Jantzen J. C., Modular representations of reductive Lie algebras, Journal of Pure and Applied Algebra 152 (2000), 133-185.

[12] Jantzen J. C., Reresentations of Algebraic Groups, 2nd edn, American Mathematical Society, Providence, RI, 2003.

[13] Jantzen J. C., Kohomologie Von p-Lie-Algebra und nilpotente Elemente, Abh. Math. Sem. Univ. Hamburg 56 (1986), 191-219.

[14] Jessen B. R., Representation Theorey of Lie algebras in prime characteristic, Progress Report, Aarhus University, 1999.

[15] Lusztig G., Periodic W-graphs, Represent. Theory 1 (1997), 207-279.

[16] Kac V. and Weisfeiler B., Coadjoint action of a semisimple algebraic group and the center of the enveloping algebra in characteristic p, Indag. Math. 38 (1976), 136-151.

Department of Mathematics, East China Normal University, Shanghai 200052, China.

E-mail address: 52050601014@student.ecnu.edu.cn

Department of Mathematics, East China Normal University, Shanghai 200062, CHINA.

E-mail address: bshu@euler.math.ecnu.edu.cn 